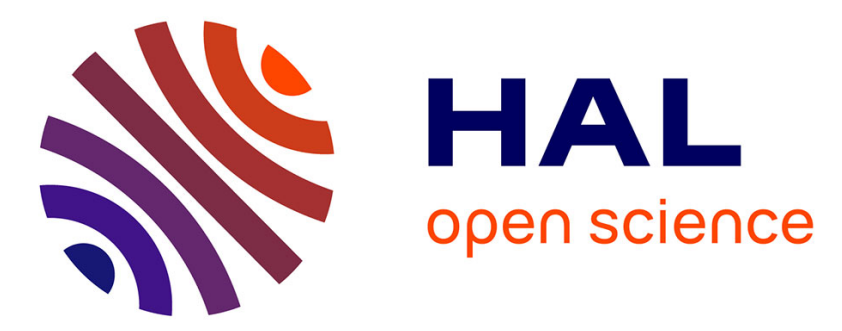

\title{
Potential-core closing of temporally developing jets at Mach numbers between 0.3 and 2: Scaling and conditional averaging of flow and sound fields
}

Christophe Bogey, Pierre Pineau

\section{- To cite this version:}

Christophe Bogey, Pierre Pineau. Potential-core closing of temporally developing jets at Mach numbers between 0.3 and 2: Scaling and conditional averaging of flow and sound fields. Physical Review Fluids, 2019, 4, 10.1103/PhysRevFluids.4.124601 . hal-02400864

\section{HAL Id: hal-02400864 \\ https://hal.science/hal-02400864}

Submitted on 9 Dec 2019

HAL is a multi-disciplinary open access archive for the deposit and dissemination of scientific research documents, whether they are published or not. The documents may come from teaching and research institutions in France or abroad, or from public or private research centers.
L'archive ouverte pluridisciplinaire HAL, est destinée au dépôt et à la diffusion de documents scientifiques de niveau recherche, publiés ou non, émanant des établissements d'enseignement et de recherche français ou étrangers, des laboratoires publics ou privés. 


\title{
Potential-core closing of temporally developing jets at Mach numbers between 0.3 and 2: Scaling and conditional averaging of flow and sound fields
}

\author{
Christophe Bogey $\odot^{*}$ and Pierre Pineau $\odot$ \\ Univ Lyon, Ecole Centrale de Lyon, INSA Lyon, Université Claude Bernard Lyon I, CNRS, \\ Laboratoire de Mécanique des Fluides et d'Acoustique, UMR 5509, F-69134, Ecully, France
}

(Received 5 July 2019; published 9 December 2019)

\begin{abstract}
In a previous work [Bogey, J. Fluid Mech. 859, 1022 (2019)], the potential-core closing of temporally developing isothermal round jets at a Mach number $M=0.9$ was shown to generate a strong axisymmetric noise component in the downstream direction. The persistence of this component is investigated in the present work for jets at $M=0.3$, $0.6,1.3$, and 2 computed by direct numerical simulation, from a low subsonic to a high supersonic Mach number. The flow and sound fields are presented, and the Mach number scaling of their magnitudes and spectral content is examined. The centerline velocity and hydrodynamic pressure spectra are close to each other using $k_{z} r_{0}$ in abscissa, where $k_{z}$ and $r_{0}$ are the axial wave number and the initial jet radius, respectively. The sound spectra for $M \leqslant 1.3$ collapse well when they are plotted as a function of $k_{z} r_{0} M^{-1}$ and adjusted in amplitude using a $M^{7.5}$ power law, whereas a $k_{z} r_{0}$ scaling and a lower power-law exponent seem to apply to the spectra for $M \geqslant 1.3$. The flow and sound fields are then correlated with each other, and conditionally averaged based on a synchronization of the fields with the minimum values of centerline velocity at potential-core closing. The noise component radiated in the downstream direction for $M=0.9$ is clearly identified for $M=0.6,1.3,2$, and is also detected, albeit with more difficulty, for $M=0.3$, indicating the presence of the associated sound source over a wide range of Mach numbers. In all cases, its generation process extracted by the conditional averaging consists in the growth of a spot of low velocity and a high vorticity level in the inner side of the mixing layer, reaching a peak intensity at its arrival on the axis and weakening subsequently. The use of different trigger conditions for the averaging suggests that, for a given Mach number, the amplitude of the acoustic waves radiated during that stage depends linearly on the velocity deficit and the strength of the vortical structures on the jet centerline at the time of potential-core closing.
\end{abstract}

DOI: 10.1103/PhysRevFluids.4.124601

\section{INTRODUCTION}

Since the early 1950s, noise generation in jet flows has been investigated in a very large amount of theoretical, experimental, and numerical studies. Sound sources have been described in several ways involving different concepts, such as acoustic quadrupoles [1], self noise and shear noise [2], vortex sound [3], instability waves [4], large-scale ordered structures [5], fine-scale turbulence [6], and wave packets [7], just to mention a few seminal papers in these fields. Significant progress has thus been made, in particular for supersonic jets [8]. Some questions, however, are still unanswered about the strong mixing-noise component prevailing in the downstream direction of jets, whose properties are quite distinct from those of the omnidirectional, broadband mixing-noise

*christophe.bogey@ec-lyon.fr 
component dominant in the upstream and sideline directions [9]. This downstream component is mainly axisymmetric [10,11], and appears to be generated by large-scale structures and/or instability waves over a wide range of Mach numbers [12], typically from $M=u_{j} / c_{0}=0.5$ up to $M=2$, where $u_{j}$ and $c_{0}$ are the jet velocity and the speed of sound in the ambient medium. On the basis of the results of noise source localizations [13-15] and flow-noise crosscorrelations [16-18], it was also found to originate at the end of the jet potential core where the shear layers merge. Its generation mechanism is most likely not to depend significantly on the Reynolds number [18-20] but is not yet clearly understood [9,11]. Some of the basic characteristics of the noise component themselves continue to be matters of debate. For instance, this is the case for the dependence of its peak frequency on the velocity. This frequency was indeed found to remain around a Strouhal number of $\mathrm{St}_{D}=f D / u_{j}=0.15$, where $f$ and $D$ are the frequency and the jet diameter, in a number of work [21,22], but scalings as the Helmholtz number $\mathrm{Hm}=$ $f D / c_{0}$, with [23] or without $[11,24]$ a correction by a Doppler factor, were also reported in the literature.

In order to get a new insight into the generation of the jet noise component radiated in the downstream direction, temporally developing subsonic axisymmetric mixing layers were simulated well after the time of their potential-core closing in a recent study [25]. Despite obvious differences with their spatially developing counterparts, temporally developing planar and axisymmetric mixing layers have been computed over the last few years to describe the turbulent development and the compressibility effects [26-29] and to explore acoustic sources in free shear flows [30-34]. In Ref. [25], two jets at a Mach number of 0.9 and at diameter-based Reynolds numbers of 3125 and 12500 were considered. The jet at a Reynolds number of 12500 develops more rapidly, exhibits more fine turbulent scales, and generates more high-frequency acoustic waves than the jet at a Reynolds number of 3125 , as expected. In both cases, however, strong low-frequency acoustic waves are emitted in the downstream direction approximately at the time of potential-core closing. These waves are dominated by the axisymmetric azimuthal mode and are strongly correlated with the centerline flow fluctuations. This led us to conditionally average the jet flow and near pressure fields using a sampling synchronization with the minimum values of centerline axial velocity at potential-core closing. In this way, it was found that a spot characterized by a lower velocity and a higher vorticity level relative to the background flow field develops in the interface region between the mixing layer and the potential core, strengthens rapidly and reaches a peak intensity when arriving on the jet axis, and then breaks down. That process is accompanied by the growth and decay of a hydrodynamic pressure wave around the jets, and by the radiation of sound waves in the downstream direction, These results, not necessarily expected at first sight, are similar to those observed for spatially developing jets, e.g., in Refs. [35-37] for the near-field wave packets. They suggest that the mechanism responsible for the jet noise component dominant in the downstream direction does not depend on the presence of a nozzle or of a potential core of finite length, which would be necessary for the establishment of feedback modes. On the contrary, it seems to be linked to the physics of the interactions and merging of parallel mixing layers, as was suggested by the calculations of two-dimensional plane jets subjected to a spatially localized initial disturbance by Sandham et al. [38]. In the latter two-dimensional simulations, however, the contra-rotative vortical structures in the two jet mixing layers mutually interact, but they cannot merge, preventing the potential-core closing.

A logical next step is now to deal with temporally developing jets at different Mach numbers, in order to examine the influence of the velocity on the noise generation at the potential-core closing. The variations of the acoustic characteristics of spatially developing jets with the Mach number have been investigated in a lot of studies in order to obtain information on sound components and their associated sources. For instance, the theoretical work of Lighthill [1] and Ffowcs Williams [39] established that the overall sound pressure level should increase with the eighth power of velocity for subsonic jets, but with the third power of velocity for supersonic jets. Overall, these power laws apply to far-field noise measurements, although the power-law exponent appears to change with the radiation angle [21]. In particular, there is a rapid increase 
of the exponent as the angle decreases [9]. Thus, as noise intensity typically varies as $M^{7.5}$ at $90^{\circ}$ relative to the jet direction [22,23], exponents as high as 10 or 11 can be obtained for sufficiently small radiation angles $[9,22]$. In the same way, using subsonic or supersonic wave filtering, Ryu et al. [40] found that the supersonic downstream-propagating components scale with the 10th power of the jet velocity in the near pressure fields of jets at Mach numbers ranging from 0.51 to 1.95 . Regarding the peak frequency in the far-field spectra, it scales with the Strouhal number $\mathrm{St}_{D}$ in the sideline direction, but there is no clear consensus for its scaling in the downstream direction, as mentioned previously in this introduction. Finally, the levels of the correlations obtained between the sound pressure fluctuations in the downstream direction and the flow fluctuations around the end of the jet potential core strongly depend on the jet velocity. Indeed, they are high for $M \simeq 2$ but sharply decrease for lower Mach numbers, falling below the noise floor for $M \leqslant 0.8$ [17], which supports the inefficiency of the corresponding acoustic source in jets at sufficiently low velocities.

Based on the above, the properties of the jet noise components radiated by four temporally developing isothermal round jets at a Reynolds number of 3125 and at Mach numbers of 0.3, 0.6, 1.3 , and 2 when their potential cores close are investigated in the present paper. For that, the jet flow and sound fields are computed by direct numerical simulation (DNS) using the same numerical setup as for the jets at $M=0.9$ in Ref. [25], up to times well after the potential-core closing. In a sense, the present study constitutes an extension of that of Freund et al. [29] in which axisymmetric mixing layers at Mach numbers ranging from 0.2 to 3.5 and at diameter-based Reynolds numbers between 4200 and 6400 were simulated by DNS until their potential-core closing. The flow fields were analyzed just before that time, and the acoustic fields were not captured due to the limited extent of the computational domain in the radial direction. In this work, the objective will be to describe the velocity and pressure fields of the jets during the potential core closing and afterwards for the first time for temporally developing jets at various Mach numbers to the best of our knowledge. The particular question that arises is whether the jets all produce axisymmetric low-frequency sound waves in the downstream direction around the time of potential-core closing, as observed for the jet at Mach 0.9. This is not obvious for the low subsonic case at $M=0.3$ given the experimental results of the literature [9,17], but also for the high supersonic case at $M=2$ for which strong Mach-wave radiation is expected. The variations with the Mach number of the results, including the magnitudes and spectral contents of the centerline velocity fields and of the hydrodynamic and acoustic pressure fields, will be examined, and compared with those available for spatially developing jets. Moreover, in order to highlight the noise generation mechanism, cross-correlations will be computed between centerline flow quantities and pressure fluctuations in the hydrodynamic and near acoustic fields of the jets. More importantly, conditional averaging will also be performed, as was, for instance, the case in the recent studies of Schmidt and Schmid [41] and Pineau and Bogey [42] to isolate the flow event precursor of Mach-wave radiation in supersonic jets. In practice, the DNS fields will be synchronized with the minimum values of centerline velocity at the time of potential-core closing, when the latter are below a given threshold. Different thresholds will moreover be used to search for connections between the emitted sound waves and the centerline flow features at core closing, including the velocity deficit and the strength of the vortical structures on the jet centerline at that time.

The paper is organized as follows. The main characteristics of the jets and of the simulations, including initial flow conditions, numerical methods, grid and computational parameters, and the procedure of linear stability analysis used in this work are documented in Sec. II. The simulation results, namely, vorticity and pressure snapshots, the key features of the jet velocity and near pressure fields, flow-noise cross-correlations and conditionally averaged flow and sound fields, are shown and commented on in Sec. III. Concluding remarks are given in Sec. IV. Finally, some results of the linear stability analysis and flow-noise correlations computed from the conditionally averaged fields are provided in two appendices. 


\section{PARAMETERS}

\section{A. Jet definition}

At initial time $t=0$, five axisymmetric, isothermal mixing layers are imposed in the computational domain. They have Mach numbers of $M=0.3,0.6,0.9,1.3$, and 2, and a Reynolds number of $\operatorname{Re}_{D}=u_{j} D / v=3125$, where $u_{j}$ and $D=2 r_{0}$ are the initial centerline velocity and diameter, and $v$ is the kinematic molecular viscosity. For the axial velocity, a hyperbolic-tangent profile of axial velocity is prescribed. Following the variations of $\delta_{\theta} / r_{0}$ with the Reynolds number observed in experiments for initially laminar jets [43], the momentum thickness of the velocity profile is fixed at $\delta_{\theta}=0.0358 r_{0}$. This leads to the initial momentum Reynolds number of $\operatorname{Re}_{\theta}=u_{j} \delta_{\theta} / v=56$, and hence to significant viscous effects in the mixing layers [44,45]. Radial and azimuthal velocities are set to zero, pressure is equal at $p_{0}$, and density is determined by a Crocco-Busemann relation.

The mixing layers are simulated well after the disappearance of their potential cores. For that reason, they will be referred to as jets in what follows, and this despite their differences with respect to spatially developing jets, in terms of flow entrainment in particular [46]. As in the DNS of Freund et al. [29], their Reynolds numbers are low in order to resolve all the flow turbulent scales using reasonable mesh spacings. A second reason for that Reynolds number value is that the noise component generated at potential-core closing was found to be more predominant in the acoustic fields of Mach 0.9 jets for $\operatorname{Re}_{D}=3125$ than for $\operatorname{Re}_{D}=12500$ and 50000 in Ref. [25], which should ease its extraction in the present study. It can also be noted that the jet at $M=2$ was considered in a previous paper [47] in a preliminary exploration of the formation of shocked waves in the vicinity of supersonic jets.

At time $t=0$, velocity disturbances of low amplitude are added in the mixing layers in order to seed the laminar-turbulent transition. For this, divergence-free Gaussian ring vortices of radius $r_{0}$ are introduced [48]. These vortices have a half-width of $2 \delta_{\theta}$, and are regularly distributed in the axial direction every $\Delta z=0.025 r_{0}$, where $\Delta z$ is the axial mesh spacing. At each position, the vortex has a maximum velocity randomly fixed between 0 and $\alpha_{\text {exc }}$ and is weighted in the azimuthal direction by the function $\cos \left(n_{\theta} \theta+\varphi\right)$ where $n_{\theta}$ and $\varphi$ are randomly chosen between 0 and 32 and between 0 and $2 \pi$, respectively. For the jets at $M=0.3,0.6,0.9$, and 1.3 , the same forcing strength of $\alpha_{\text {exc }}=0.01 u_{j}$ is used. It was chosen arbitrarily to provide a peak turbulence intensity close to $1 \%$ at $t=0$. For the jet at $M=2$, for which the growth rates of the linear instability waves are much weaker than for the jets at lower Mach numbers as shown in Sec. III B, such an initial condition was found to lead to a laminar-turbulent transition process much longer than $100 r_{0} / u_{j}$, which would be very costly. The forcing strength is therefore of $\alpha_{\mathrm{exc}}=0.04 u_{j}$, resulting in a turbulence intensity of $4 \%$ at $t=0$ in that case. The effects of the forcing strength on the early development of the instability waves were examined for a jet at $M=2$ and $\operatorname{Re}_{D}=12500$ in Pineau and Bogey [42]. Except for the amplitude, very similar results were found for $\alpha_{\mathrm{exc}}=0.0025 u_{j}, 0.01 u_{j}$, and $0.04 u_{j}$ at $t=5 r_{0} / u_{j}$. Therefore, for the Mach 2 jet of the present study, it is most likely that the flow properties when the potential core closes, that is around $t=50 r_{0} / u_{j}$, do not significantly depend on the forcing strength. Finally, several runs are performed for each jet using different random seeds in order to improve the statistical convergence of the results.

\section{B. Numerical methods}

The numerical framework is identical to that used in recent simulations of spatially developing [49-51] and temporally developing [25,42] round jets. The simulations are carried out using an in-house solver of the three-dimensional filtered compressible Navier-Stokes equations in cylindrical coordinates $(r, \theta, z)$ based on low-dissipation and low-dispersion, high-order explicit schemes. The axis singularity is taken into account by the method of Mohseni and Colonius [52]. In order to alleviate the time-step restriction near the cylindrical origin, the derivatives in the azimuthal direction around the axis are calculated at coarser resolutions than permitted by the grid [53]. For the points closest to the jet axis, they are evaluated using 16 points, yielding an effective resolution 
TABLE I. Jet Mach number $M$, number of runs $n_{\text {runs }}$, extents of the computational domain in the axial and radial directions $L_{z}$ and $L_{r}$, simulation time $t_{\max }$, and time of potential-core closing $t_{c}$.

\begin{tabular}{lrcccc}
\hline \hline$M$ & $n_{\text {runs }}$ & $L_{z} / r_{0}$ & $L_{r} / r_{0}$ & $t_{\max } r_{0} / u_{j}$ & $t_{c} r_{0} / u_{j}$ \\
\hline 0.3 & 5 & 240 & 30 & 50 & 16.9 \\
0.6 & 5 & 240 & 30 & 60 & 18.5 \\
0.9 & 10 & 120 & 30 & 75 & 21.6 \\
1.3 & 5 & 240 & 30 & 75 & 30.1 \\
2 & 4 & 240 & 13 & 96 & 48.9 \\
\hline \hline
\end{tabular}

of $2 \pi / 16$. Fourth-order 11-point centered finite differences are used for spatial discretization, and a second-order six-stage Runge-Kutta algorithm is implemented for time integration [54]. A 12th-order 11-point centered filter is applied explicitly to the flow variables every time step in order to remove grid-to-grid oscillations while leaving larger scales mostly unaffected. Noncentered finite differences and filters are also used near the grid boundaries [55,56]. Radiation conditions [57,58] are applied at the sideline boundaries to avoid significant acoustic reflections. Finally, periodic boundary conditions are imposed in the axial direction. Given the size of $L_{z}=120 r_{0}$ or $240 r_{0}$ of the computational domains, they are unlikely to distort the jet flow development or to impose artificial modes in that direction. This is demonstrated for the jet at $M=0.9$ in Ref. [25] on the basis of velocity correlation functions.

\section{Simulation parameters}

The present simulations are fully resolved DNS, which was checked by calculating the different terms of the turbulent kinetic energy budget and making sure their sum is nil. Their main parameters are provided in Table I. The grids all contain 980 million points, with $n_{r}=382, n_{\theta}=512$ and $n_{z}=4800$ for the jet at $M=0.9, n_{r}=382, n_{\theta}=256$, and $n_{z}=4800$ for $M=0.3,0.6$, and 1.3, and $n_{r}=382, n_{\theta}=256$, and $n_{z}=9600$ for $M=2$. They extend up to $L_{z}=120 r_{0}$ and out to $L_{r}=30 r_{0}$ in the first case, $L_{z}=240 r_{0}$ and $L_{r}=30 r_{0}$ in the second one, and $L_{z}=240 r_{0}$ and $L_{r}=13 r_{0}$ in the third one. The mesh spacing in the axial direction is uniform and equal to $\Delta z=0.025 r_{0}$, whereas the mesh spacing in the radial direction varies. The latter is minimum and equal to $\Delta r=0.006 r_{0}$ at $r=r_{0}$. It is maximum and equal to $\Delta r=0.2 r_{0}$ for $r \geqslant 16 r_{0}$ for the jets at $M \leqslant 1.3$ and to $\Delta r=0.05 r_{0}$ for $r \geqslant 4 r_{0}$ for the jet at $M=2$, yielding wave numbers of $k_{z} r_{0}=7.8$ and 31, respectively, for a wave discretized by four points per wavelength. The mesh is finer for the Mach 2 jet than for the others in order to allow for the propagation of shocked waves in the near field of the former jet [42]. The use of $n_{\theta}=256$ and 512 points in the azimuthal direction leads to $r \Delta \theta=0.024 r_{0}$ and $0.012 r_{0}$ at $r=r_{0}$.

The computations are performed using an OpenMP-based in-house solver on 32-core nodes of Intel E5-4650 processors with a clock speed of $2.7 \mathrm{GHz}$ and 16-core nodes of Intel E5-2670 processors at $2.6 \mathrm{GHz}$. The total number of iterations is equal to 18000 for $M=0.3$ and 0.6 , to 23000 for $M=0.9$, to 17000 for $M=1.3$ and to 21000 for $M=2$, leading to the final times $t_{\max }$ of $50 r_{0} / u_{j}$ for $M=0.3$, of $60 r_{0} / u_{j}$ for $M=0.6$, of $75 r_{0} / u_{j}$ for $M=0.9$ and 1.3 , and of $96 r_{0} / u_{j}$ for $M=2$. After preliminary tests of numerical stability, the value of the time step $\Delta t$ was chosen so that $c_{0} \Delta t / \Delta r\left(r=r_{0}\right)=1.5,0.9,0.6,0.55$, and 0.37 for $M=0.3,0.6,0.9,1.3$, and 2 , respectively. During the simulations, density, the three velocity components, pressure and vorticity norm are recorded every $0.1 r_{0} / u_{j}$ on the jet axis and on the cylindrical surfaces at $r=r_{0}, 4 r_{0}$, and $20 r_{0}$, and every $0.2 r_{0} / u_{j}$ on the four azimuthal planes at $\theta=0, \pi / 2, \pi$, and $3 \pi / 2$. Their Fourier coefficients in the azimuthal directions, estimated over the full section $(r, z)$, are also saved for the first four azimuthal modes every $0.2 r_{0} / u_{j}$. For each jet, several runs, namely, 10 runs for $M=0.9$, five runs for $M=0.3,0.6$, and 1.3 , and four runs for $M=2$, are executed using different random seeds. The statistical results obtained in each run are averaged over the periodic directions $z$ and $\theta$, and are then 
ensemble averaged, providing mean values denoted by $\langle$.$\rangle . Their convergence for the jet at M=0.9$ is shown in Ref. [25].

\section{Linear stability analysis}

Inviscid temporal stability analyses have been carried out directly from the DNS profiles of mean axial velocity and mean density obtained at $t=0$ and between $t=t_{c}-15 r_{0} / u_{j}$ and $t=t_{c}$, where $t_{c}$ is the time of potential-core closing defined hereafter, using the same procedure as in recent study on spatially developing subsonic jets [51]. For simplicity's sake, viscous effects are not taken into account, although they are expected to be significant at the low Reynolds numbers $\operatorname{Re}_{\theta}$ considered in this work $[44,45]$. In practice, for a given time $t$ and a given axial wave number $k_{z}$, the mean profiles of axial velocity and density are considered as if they were stationary and their instability modes are calculated numerically by solving the compressible Rayleigh equation [59-61]. These instabilities are computed through a shooting technique [45] based on the Euler method for the integration step and on the secant method for the search of the complex angular frequency $\omega$. The integration is performed on a grid with a spatial step of $0.0001 r_{0}$, extending from the DNS grid point closest to the jet axis out to $r=5 r_{0}$.

\section{RESULTS}

\section{A. Vorticity and pressure snapshots}

Fields of vorticity norm and pressure fluctuations obtained for $M=0.3,0.6,1.3$, and 2 in the $(z, r)$ plane at $t=t_{c}$ and $t_{c}+18 r_{0} / c_{0}$, where $t_{c}$ denotes the time when the mean centerline velocity is equal to $0.95 u_{j}$, are represented in Figs. 1 and 2. Their time evolutions are shown in the corresponding movies available as Supplemental Material [62]. Fields reconstructed from the first four azimuthal modes in the $(r-\theta)$ plane at $z=0$ are also provided in the figures. Note that the pressure fluctuations are normalized by $p_{0} M^{2}$ at $t=t_{c}$ and by $p_{0} M^{3.75}$ at $t_{c}+18 r_{0} / c_{0}$, according to the respective Mach number scalings of the levels of the hydrodynamic and acoustic pressure fluctuations that will be found in Sec. III C.

For all jets, instability waves grow at earlier times in the initially laminar hyperbolic-tangent velocity profiles [59], leading to the rolling up of vortices in the mixing layers. As the Mach number increases, as in the DNS of Freund et al. [46], this occurs more rapidly, and the vortices are weaker and more elongated in the axial direction, in particular for the jet at a Mach number of 2 . The vortices develop and interact with each other through the jet potential core at $t=t_{c}$ in Fig. 1 . Then the mixing layers merge on the jet axis, resulting in the disappearance of the potential core and the presence of vortical structures in the whole radial section; see, for instance, Fig. 2.

In the jet near fields, large-scale pressure waves are first seen in the immediate vicinity of the flow. They correspond to the hydrodynamic pressure disturbances induced by the large turbulent structures of the shear layers $[63,64]$. They are well visible at $t=t_{c}$ in Fig. 1. At that time, acoustic waves also start to emerge from the jet flow, especially for the jet at $M=2$ for which Mach waves are generated by the supersonic motion of the flow structures in the mixing layers [47]. The acoustic waves appear more clearly at $t=t_{c}+18 r_{0} / c_{0}$ in Fig. 2 as they propagate farther from the axis. In all cases, they travel mainly in the downstream direction, as observed at shallow angles for spatially developing subsonic jets $[48,65]$. They are mostly symmetric with respect to the jet centerline [see also their three-dimensional geometries in the $(z-\theta)$ planes] and have a large spatial extent along the wave front direction. Their levels strongly increase with the Mach number, as expected, and range approximately from $5 \mathrm{~Pa}$ at $M=0.3$ up to $3000 \mathrm{~Pa}$ at $M=2$. In addition, their associated wavelengths decrease with the jet velocity. For instance, they are typically equal to $20 r_{0}$ at $M=0.6$ and to $10 r_{0}$ at $M=1.3$.

\section{B. Properties of the velocity fields}

In order to characterize the linear instability waves growing initially in the laminar shear layers, the instability amplification rates $\operatorname{Im}(\omega) r_{0} / u_{j}$ obtained for the first two azimuthal modes $n_{\theta}=0$ 

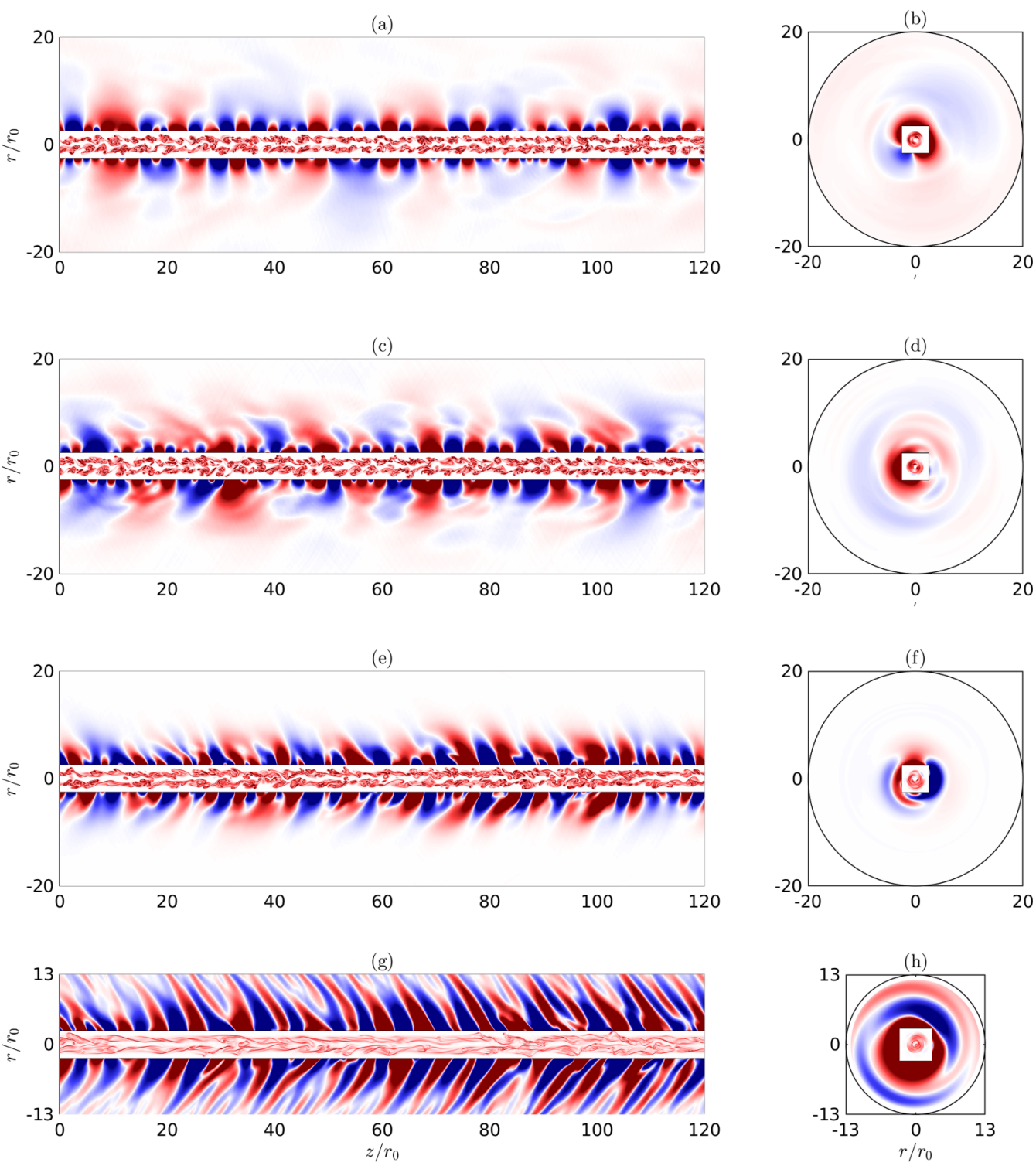

FIG. 1. Vorticity norm for $r \leqslant 2.5 r_{0}$ and pressure fluctuations otherwise, obtained at $t_{c}$ for $M=0.3,0.6$, 1.3, and 2, from top to bottom: (left) full fields in the $(r-z)$ section, (right) fields reconstructed from the first four azimuthal modes at $z=0$. The color scales range between $\pm 5 u_{j} / r_{0}$ and $\pm 0.002 p_{0} M^{2}$, from blue to red. See also the movies available as Supplemental Material [62].

and $n_{\theta}=1$ from the flow profiles at $t=0$ using the methodology described in Sec. II D are represented in Figs. 3(a) and 3(b) as a function of the axial wave number $k_{z} r_{0}$. For $M=0.3$, the curves for $n_{\theta}=0$ and 1 are very similar to each other, and to that given by the temporal linear stability analysis of Michalke [59] for an incompressible two-dimensional mixing layer with a hyperbolic-tangent velocity profile, which yields a peak value of $\operatorname{Im}(\omega) r_{0} / u_{j}=1.34$ at $k_{z} r_{0}=1.21$ using the parameters of the present mixing layers. With increasing Mach number, the amplification rates are reduced, the range of unstable wave numbers is narrower, and the mode $n_{\theta}=1$ becomes more unstable than the axisymmetric mode, in agreement with the literature [60]. 
(a)

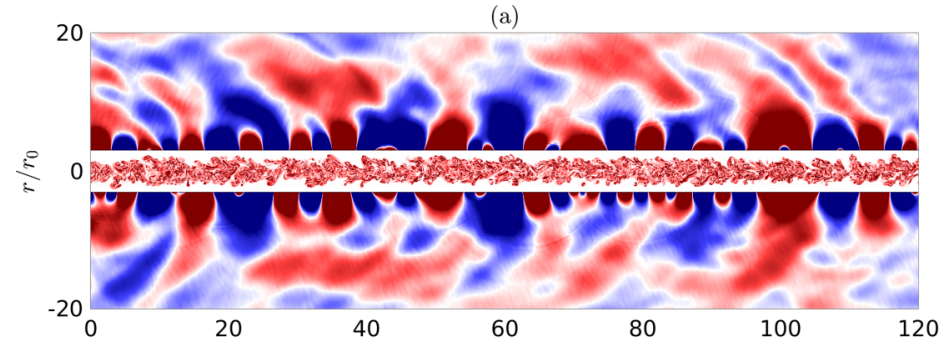

(c)

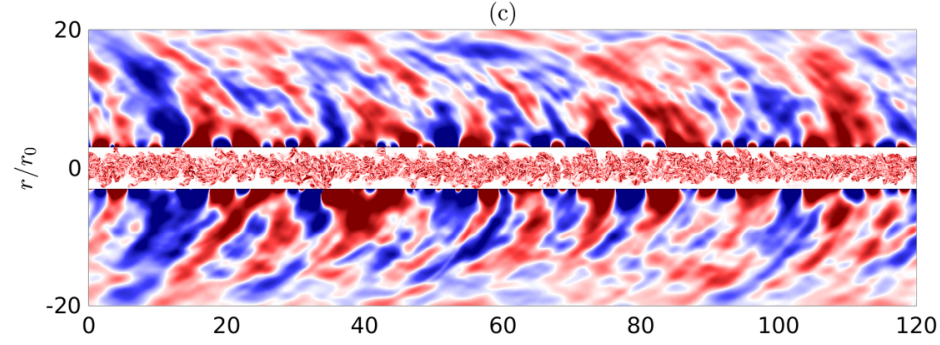

(e)

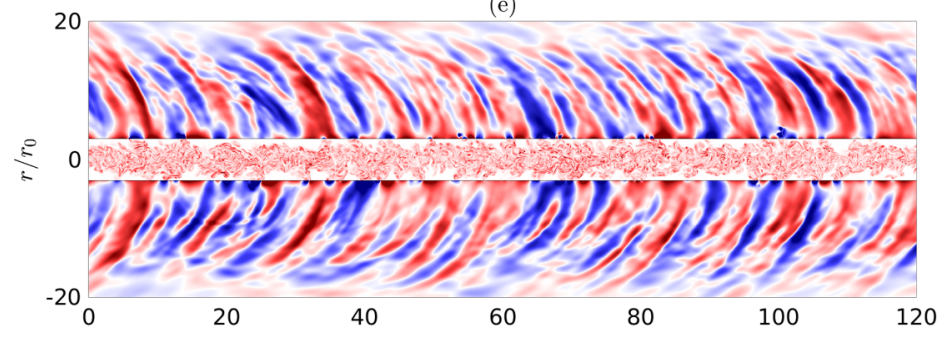

(g)

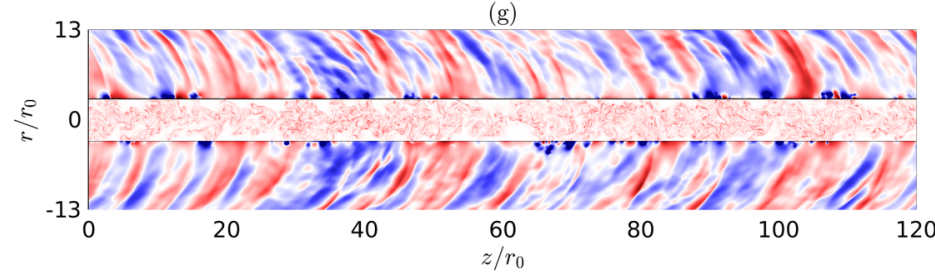

(b)

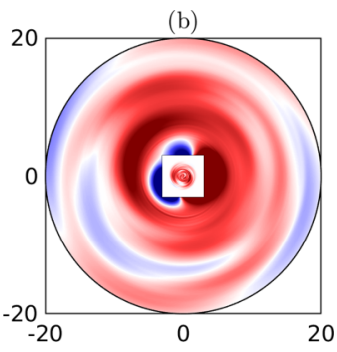

(d)
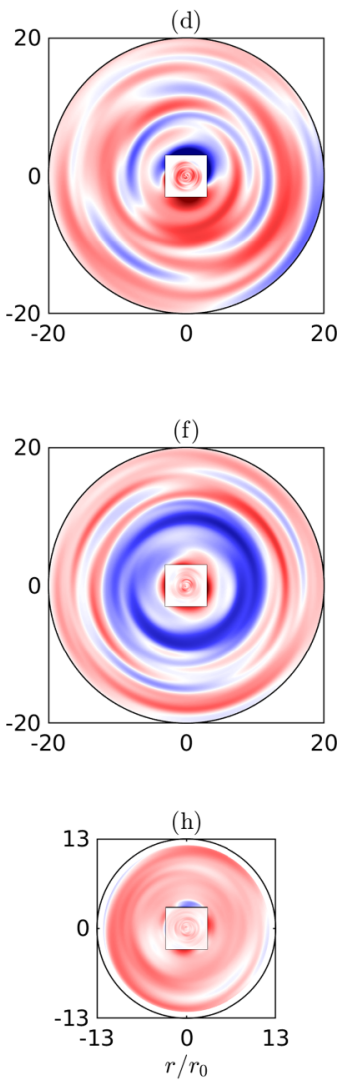

FIG. 2. Vorticity norm for $r \leqslant 3 r_{0}$ and pressure fluctuations otherwise, obtained at $t_{c}+18 r_{0} / c_{0}$ for $M=$ $0.3,0.6,1.3$, and 2 , from to bottom: (left) full fields in the $(r-z)$ section, (right) fields reconstructed from the first four azimuthal modes at $z=0$. The color scales range between $\pm 5 u_{j} / r_{0}$ and $\pm 0.003 p_{0} M^{3.75}$, from blue to red. See also the movies available as Supplemental Material [62].

The spectra of axial velocity fluctuations normalized by the initial centerline velocity, calculated at $r=r_{0}$ at $t=2 r_{0} / u_{j}$ for $M=0.3,0.6,0.9$, and 1.3 and at $t=8 r_{0} / u_{j}$ for $M=2$ are plotted in Fig. 3(c). The spectrum for $M=2$ is shown at a later time in order to obtain components of comparable levels in the five cases despite the much weaker amplification rates at $M=2$ than at lower Mach numbers. Overall, the results are consistent with the linear stability analysis. In particular, the peak wave numbers in the spectra are just slightly lower than the most unstable wave numbers predicted by the analysis. For the jets at $M \leqslant 1.3$ at $t=2 r_{0} / u_{j}$, in addition, the fluctuation intensities decrease with the Mach number, as expected. 
(a)

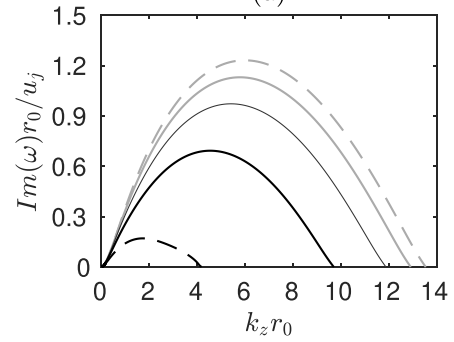

(b)

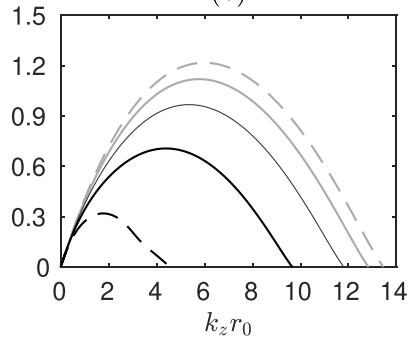

(c)

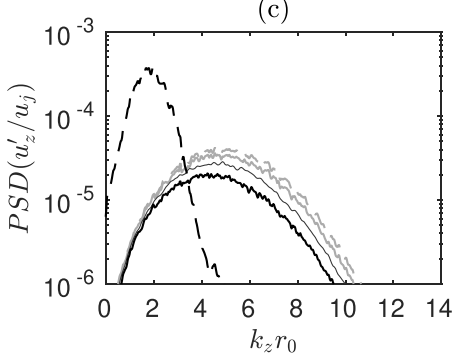

FIG. 3. Instability growth rates obtained at $t=0$ for (a) $n_{\theta}=0$ and (b) $n_{\theta}=1$, and (c) power spectral densities (PSDs) of axial velocity fluctuations at $r=r_{0}$ at $t=2 r_{0} / u_{j}$ for $M \leqslant 1.3$ and at $t=8 r_{0} / u_{j}$ for $M=2$, as a function of axial wave number: $---M=0.3, \longrightarrow M=0.6, \longrightarrow M=$ $1.3,--M=2$.

The variations of the shear-layer momentum thickness are presented as a function of $t u_{j} / r_{0}$ in Fig. 4(a). In accordance with the results above, the shear layer spreads more slowly at a higher Mach number. As a result, the jet potential core persists over a longer time period, and the mean centerline velocity reaches a value of $0.95 u_{j}$ at a time $t_{c}$ ranging from $16.9 r_{0} / u_{j}$ for $M=0.3$ up to $48.9 r_{0} / u_{j}$ for $M=2$, as reported in Table I. At that time, the momentum thickness is nearly the same for all cases and is close to $\delta_{\theta}=0.24 r_{0}$. For the comparison, most of the results in the paper of Freund et al. [29] are presented for $\delta_{\theta}=0.2 r_{0}$, which is slightly before $t=t_{c}$.

In order to examine the differences in mean flow development after the potential-core closing, the mean centerline axial velocities and the jet half-widths are plotted in Figs. 4(b) and 4(c) as a function of $\left(t-t_{c}\right) u_{j} / r_{0}$. The curves obtained for $M=0.3$ and 0.6 are superimposed. The other curves deviate from them, almost imperceptibly for $M=0.9$, appreciably for $M=1.3$ and strongly for $M=2$. The velocity decay and the flow spreading are therefore weaker as the jet velocity increases, especially for Mach numbers well above 1, which can be attributed to compressibility effects [66]. Furthermore, it can be remarked that in all cases the jet half-width decreases and reaches a minimum value around $t=t_{c}$, indicating a narrowing of the axisymmetric mixing layers when the turbulent structures burst into the centerline region.

The time variations of the axial turbulence intensities at $r=0$ are displayed in Fig. 5(a). As for the mean centerline velocity in Fig. 4(b), the results are similar for the jets at $M \leqslant 1.3$, but differ for $M=2$. For $M \leqslant 1.3$, the turbulence intensities reach peaks approximately of $17 \%$ at $t \simeq t_{c}+4 r_{0} / u_{j}$, whereas for $M=2$ the peak value is obtained later, at $t=t_{c}+8.1 r_{0} / u_{j}$, and is equal to $19.4 \%$. Moreover, after the peaks, the decrease of the turbulent intensities is less pronounced

(a)

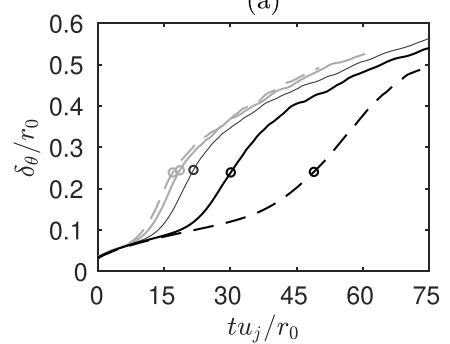

(b)

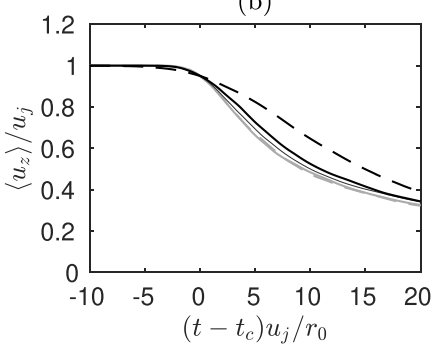

(c)

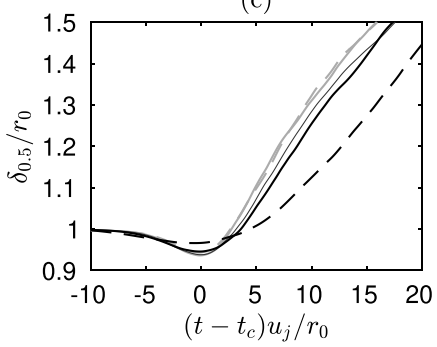

FIG. 4. Time variations of (a) the shear-layer momentum thickness, (b) the mean axial velocity at $r=0$, and (c) the jet half-width: $---M=0.3$, $M=0.6$, $M=0.9$, $M=1.3$,

$M=2$; $\circ$ momentum thickness at $t=t_{c}$. 
(a)

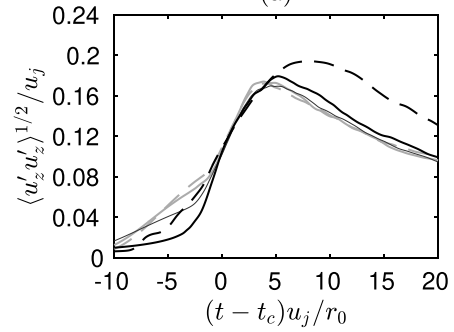

(b)

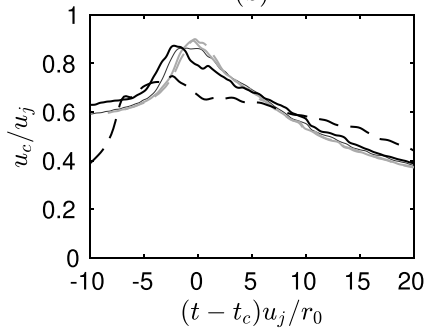

(c)

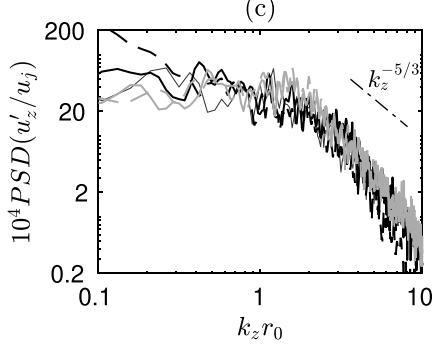

FIG. 5. Flow field at $r=0$ : time variations of (a) the rms values of axial velocity fluctuations and (b) the convection velocity, and (c) power spectral densities of velocity at $t_{c}$ as a function of axial wave number: $--M=0.3$ $M=0.6$ $M=0.9$, $M=1.3,--M=2 ; k_{z}^{-5 / 3}$.

for the jet at $M=2$ than for the others, in agreement with the slower flow development in the high-supersonic case.

The convection velocities on the axis, calculated from the correlation functions of the axial velocity fluctuations, are shown in Fig. 5(b). For $M \leqslant 1.3$, they are close to $0.6 u_{j}$ at $t \simeq t_{c}-$ $10 r_{0} / u_{j}$, then increase and reach maximum values around $0.85 u_{j}$ near the time of potential-core closing, and finally diminish. As in spatially developing jets [18], these results suggest that the turbulent structures that enter in the potential core are accelerated, which may generate sound [67]. For $M=2$, the convection velocities are lower compared to those at lower Mach numbers, and the peak values are obtained earlier, at $t \simeq t_{c}-5 r_{0} / u_{j}$.

Finally, to get an insight into the flow structures at the potential-core closing, the spectra of the centerline axial velocity fluctuations at $t=t_{c}$ are represented in Fig. 5(c) as a function of $k_{z} r_{0}$. For the comparison, a line given by the $k_{z}^{-5 / 3}$ law is also drawn. As in the mixing layers of Freund et al. [29] at the $\delta_{\theta}=0.2 r_{0}$ point, all spectra are broadband with no significant discrete peak. In addition, they do not strongly differ from each other. As the Mach number increases, however, the magnitude of the flow components tends to increase for $k_{z} r_{0} \leqslant 0.3$ and to decrease for $k_{z} r_{0} \geqslant 0.8$. Therefore, at a higher velocity, the flow structures are more elongated in the axial direction, as previously noted in the vorticity fields of Fig. 1 . This trend is consistent with the linear stability analysis conducted between $t=t_{c}-15 r_{0} / u_{j}$ and $t_{c}$ for the axisymmetric mode, reported in Appendix A, predicting lower unstable wave numbers at higher Mach numbers. This analysis also reveals no unstable wave number around the time of potential-core closing. Indeed, the last unstable wave numbers are found between $t_{c}-1.5 r_{0} / u_{j}$ for $M=0.3$ and $t_{c}-13 r_{0} / u_{j}$ for $M=2$, and are equal to $k_{z} r_{0} \simeq 0.45$ for $M=0.3,0.6$ and 0.9 , and $k_{z} r_{0} \simeq 0.35$ for $M=1.3$ and 2. It can be remarked that these wave numbers do not emerge in the spectra of Fig. 5(c).

\section{Properties of the pressure fields}

In order to examine the properties of the hydrodynamic pressure fields, clearly appearing around the jet flows at $t=t_{c}$ in Fig. 1, the pressure fluctuations are first considered at $r=2.5 r_{0}$. Indeed, for the temporally developing axisymmetric mixing layers of Freund et al. [29] with Mach numbers between 0.2 and 1.92, the rms values of pressure fluctuations at that position just before the potential-core closing scale with the square of the velocity. The values obtained for the present jets are presented as a function of $\left(t-t_{c}\right) u_{j} / r_{0}$ in Fig. 6(a), normalized by $M^{2}$. As for the centerline velocity fluctuations in Fig. 5(a), the profiles are nearly superimposed for $M \leqslant 0.9$, and differences are observed for $M=1.3$ and especially for $M=2$. At $t=t_{c}$, however, the levels are very close to each other in all cases, and similar to that approximately of 0.008 found slightly before $t_{c}$ for the mixing layers of Freund et al. [29] mentioned above. This demonstrate the hydrodynamic nature of the pressure fluctuations. For $t<t_{c}$, the levels are stronger as the Mach number increases. This can be due to the fact that, at a given $t-t_{c}$, the momentum thickness is larger, i.e., the flow is 

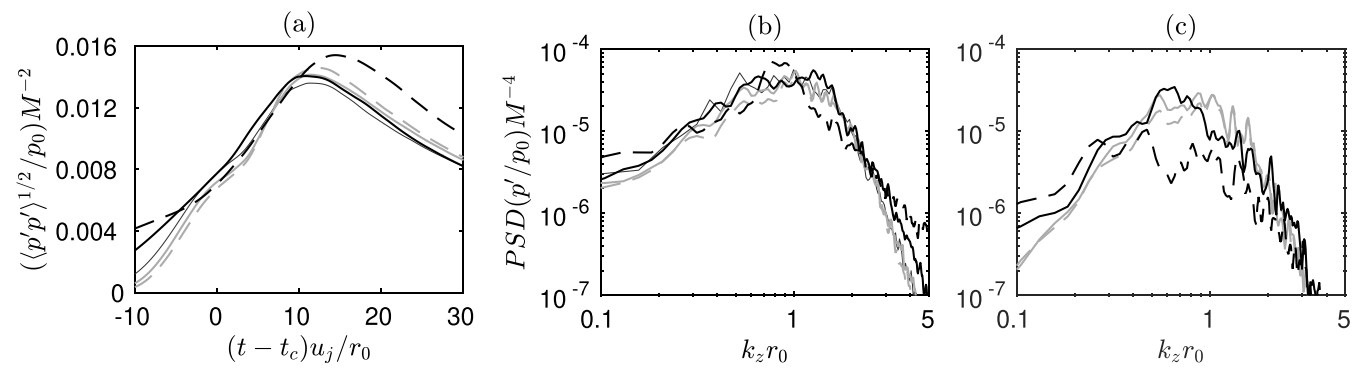

FIG. 6. Pressure field at $r=2.5 r_{0}$ : (a) time variations of the rms values of fluctuations, and power spectral densities of fluctuations obtained at $t_{c}$ from (b) the full signal and (c) mode $n_{\theta}=0$, as a function of axial wave number: $---M=0.3$, $M=0.6$, $M=0.9$, $M=1.3,--M=2$.

more developed, at a higher Mach number, refer to Fig. 4(a). For $t>t_{c}$, peaks of comparable amplitudes are reached at $t \simeq t_{c}+10 r_{0} / u_{j}$ for $M \leqslant 0.9$, that is $6 r_{0} / u_{j}$ after the time of peak turbulence intensities on the axis. For $M=2$, the maximum values are somewhat higher and are found later, around $t_{c}+12 r_{0} / u_{j}$.

The spectra estimated at $r=2.5 r_{0}$ at time $t_{c}$ for the full pressure signals and for mode $n_{\theta}=0$ are represented in Figs. 6(b) and 6(c) as a function of $k_{z} r_{0}$. They are multiplied by $M^{-4}$ to take into account the variations of the rms levels with $M^{2}$. In Fig. 6(b) the spectra resemble each other over the whole range of Mach numbers considered. This is also the case in Fig. 6(c) for $M \leqslant 1.3$, but noticeable discrepancies appear for $M=2$, in particular in terms of amplitude. This can be related to the fact that $46.6 \%, 47.7 \%$, and $34.6 \%$ of the energy are contained in the axisymmetric mode for $M=0.3,0.6$, and 1.3 , but only $14.8 \%$ for $M=2$. This reduction of the relative importance of the axisymmetric mode in the hydrodynamic pressure field with the Mach number likely results from the predominance of higher modes of instability waves at supersonic Mach numbers [42]. In terms of spectral content, the spectra peak around $k_{z} r_{0}=0.8$ for $M \leqslant 1.3$, yielding a wavelength $\lambda \simeq 8 r_{0}$. With increasing jet velocity, the pressure components are stronger at wave numbers $k_{z} r_{0} \leqslant 0.2$ and weaker at $k_{z} r_{0} \geqslant 0.8$. These trends are in agreement with those noted in the jet flow, see in Fig. 5(c).

The near acoustic fields are analysed at $r=10 r_{0}$, where sound waves prevail at $t_{c}+18 r_{0} / c_{0}$ in Fig. 2. The rms values of pressure fluctuations at that position are presented in Figs. 7(a) and 7(b). They are displayed as a function of $\left(t-t_{c}\right) c_{0} / r_{0}$ by assuming a wave propagation at the ambient speed of sound, and they are multiplied, following the classical noise scaling associated with the acoustical analogy of Lighthill [1], by $M^{-4}$ in Fig. 7(a) and, more arbitrarily, by $M^{-3.75}$ in Fig. 7(b). In all cases, the peak values are obtained at $t \simeq t_{c}+15 r_{0} / c_{0}$, suggesting that they are due to sound sources emitting at same times relative to the potential-core closing. Regarding their amplitudes,
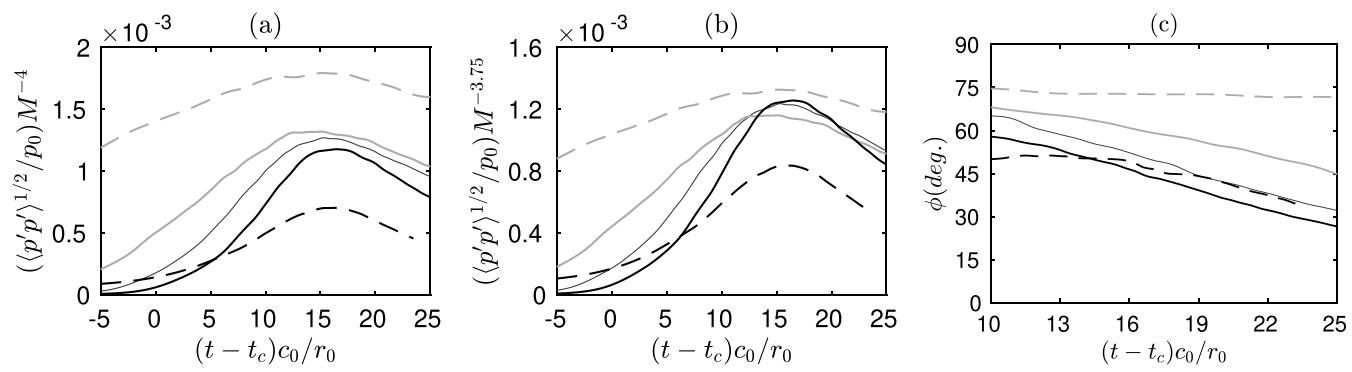

FIG. 7. Pressure field at $r=10 r_{0}$ : time variations of (a), (b) the rms values of fluctuations, and (c) the radiation angle relative to the jet direction: $=--M=0.3$, $M=0.6$, $M=0.9$

$M=1.3,--M=2$. 

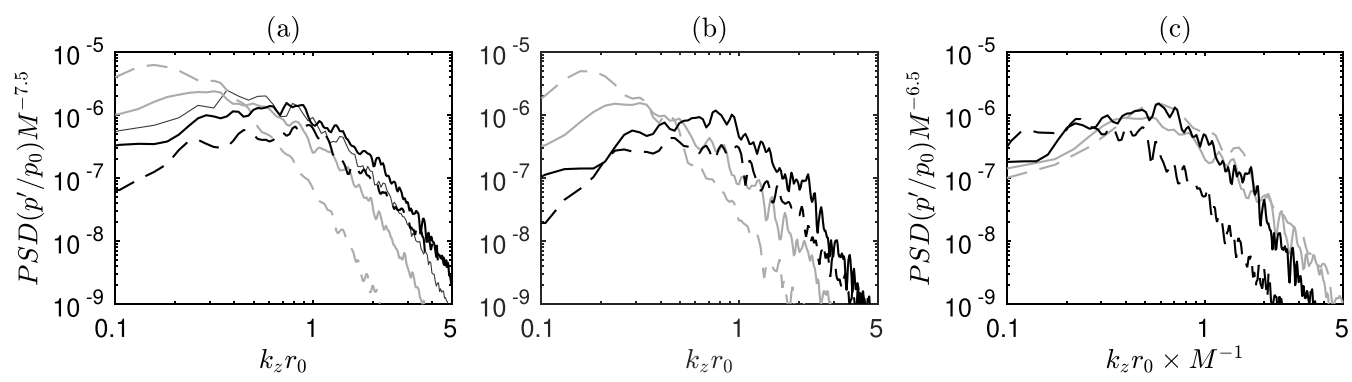

FIG. 8. Pressure field at $r=10 r_{0}$ : power spectral densities of fluctuations obtained at $t_{c}+15 r_{0} / c_{0}$ from (a) the full signal and (b) and (c) $n_{\theta}=0$, as a function of (a) and (b) $k_{z} r_{0}$ and (c) $k_{z} r_{0} \times M^{-1}:-{ }_{-}-M=0.3$, $M=0.6, \longrightarrow M=0.9, \longrightarrow M=1.3,--M=2$.

a better match is found in Fig. 7(b) than in in Fig. 7(a). In the former figure, they are similar to each other for $M \leqslant 1.3$. The sound levels thus vary roughly as $M^{7.5}$. This velocity exponent is slightly lower than the exponent of 8 predicted for subsonic jets by the analogy. More surprisingly, compared to the velocity exponents obtained for spatially developing subsonic jets, it corresponds to that measured at $90^{\circ}$ relative to the flow direction [22,23], but differs from those for small radiation angles $[9,22,40]$. This suggests that the velocity exponent typically of 10 found in the latter case is related to the spatial development of the jet flow. Last, the peak for $M=2$ is significantly below the other ones, implying that, if any, the power law exponent is lower for the noise emitted in this supersonic case, as expected given the further developments of the analogy [39].

The variations of the mean radiation angle at $r=10 r_{0}$, estimated from cross-correlations of the pressure fluctuations, are plotted in Fig. 7(c). The angle decreases monotonically with time, as the distance to the sound sources increases. For $M=2$, however, it remains near the angle $\phi=$ $\cos ^{-1}\left(c_{0} / u_{c}\right)=54^{\circ}$, predicted for Mach waves by taking the instability-wave phase velocity of $0.85 u_{j}$ obtained in Appendix A as convection velocity, between $t \simeq t_{c}+10 r_{0} / c_{0}$ and $t_{c}+16 r_{0} / c_{0}$. Moreover, the mean radiation angle is smaller at a higher Mach number, as can be seen in Fig. 2 .

Finally, the spectra calculated from the full pressure signals and from mode $n_{\theta}=0$ at $r=10 r_{0}$ at $t_{c}+15 r_{0} / c_{0}$, near the times of maximum sound level, are provided in Figs. 8(a) and 8(b) as a function of $k_{z} r_{0}$. Given the rms profiles of Fig. 7(c), they are multiplied by $M^{7.5}$. In all cases, the axisymmetric mode dominates $[10,11]$ and contains between $57.5 \%$ of the total energy for $M=0.3$ and $47.3 \%$ for $M=2$. As a result, Figs. 8(a) and 8(b) show very similar features. Focusing on mode $n_{\theta}=0$, the spectra for $M \leqslant 1.3$ clearly differ in Fig. 8(b), their strongest components shifting, for instance, from $k_{z} r_{0} \simeq 0.15$ for $M=0.3$ up to $k_{z} r_{0} \simeq 0.8$ for $M=1.3$. On the contrary, when they are plotted as a function of $k_{z} r_{0} M^{-1}$ in Fig. 8(c), they are close to each other and all peak around $k_{z} r_{0} M^{-1}=0.55$. This result is consistent with the pressure fields of Fig. 2 and suggests an acoustic radiation at a constant Strouhal number $\mathrm{St}=f D / u_{j}=0.175$, which is approximately the value measured in the downstream direction for subsonic jets [21,22]. Regarding the spectrum for $M=2$, it is not aligned with the other ones in Fig. 8(c). It has stronger low-wave-number components, and even looks like the spectrum for $M=1.3$ when depicted as a function of $k_{z} r_{0}$ in Fig. 8(b). Thus, a Helmholtz-number scaling may apply to the acoustic spectra for $M \geqslant 1.3$. Such a scaling is usually attributed to the noncompactness of the source [11], which is certainly encountered in the present high-velocity jets for which the velocity spectra at $t_{c}$ peak at lower wave numbers than the acoustic spectra.

\section{Flow-noise cross-correlations}

Correlations are computed between the flow and sound fields, as was done in numerous experimental and numerical investigations for spatially developing [16-18,68-71] and temporally developing [25] jets, in order to identify links between source and observer points. In this work, 
two types of correlations, denoted by $\mathcal{C}$ in a dimensional form and $\mathcal{R}$ in a normalized form, are calculated for the axisymmetric mode. The first ones are correlations between pressure fluctuations at $r_{\mathrm{ac}}=10 r_{0}$ in the acoustic field and flow fluctuations at $r_{\mathrm{axis}}=0$. For the axial velocity $u_{z}^{\prime}$, they are given by

$$
\mathcal{C}(\delta z, \delta t)=\left\langle p^{\prime}\left(r_{\mathrm{ac}}, \theta, z, t_{\mathrm{ac}}\right) u_{z}^{\prime}\left(r_{\mathrm{axis}}, z+\delta z, t_{\mathrm{ac}}+\delta t\right)\right\rangle,
$$

where $t_{\mathrm{ac}}=t_{c}+22 r_{0} / c_{0}, \delta z$ is the spatial separation and $\delta t$ is the time shift. The second ones are correlations between pressure fluctuations at $r_{\text {ac }}$ and at $r_{\text {hydro }}=2.5 r_{0}$, defined by

$$
\mathcal{C}(\delta z, \delta t)=\left\langle p^{\prime}\left(r_{\mathrm{ac}}, \theta, z, t_{\mathrm{ac}}\right) p^{\prime}\left(r_{\mathrm{hydro}}, \theta, z+\delta z, t_{\mathrm{ac}}+\delta t\right)\right\rangle .
$$

The normalized correlations obtained between $p^{\prime}$ at $r=10 r_{0}$ and velocity and vorticity fluctuations $u_{z}^{\prime}$ and $|\vec{\omega}|^{\prime}$ at $r=0$ for $M=0.3,0.6,1.3$ and 2 are shown in Figs. 9 (left) and 9 (middle) as a function of $\delta z / r_{0}$ and $\delta t c_{0} / r_{0}$. The dotted, solid and dashed lines reflect, respectively, the time of potential-core closing, a propagation at the ambient speed of sound between the two points considered, and a velocity of $0.65 u_{j}$.

For $M=0.3$, strong correlations are visible in Figs. 9(a) and 9(b). However, at the time of potential-core closing, they are not consistent with a propagation of acoustic waves. In order to clarify this point, correlations are calculated between pressure fluctuations at $r=25 r_{0}$ at $t=$ $t_{c}+55 r_{0} / u_{j}$ and velocity and vorticity fluctuations at $r=0$ at $t+\delta t$. They are represented in Fig. 10, in a normalized form. The levels are significant for distances $\delta z \simeq-5 r_{0}$ for all time delays for $u_{z}^{\prime}$ and for $\delta t c_{0} / r_{0} \leqslant 40$ for $|\vec{\omega}|^{\prime}$, in the same way as in Figs. 9(a) and 9(b). These correlations can be attributed to the presence of components of hydrodynamic nature in the pressure signals. Such components can indeed be expected for $k_{z} r<2$ [40,64], yielding $k_{z} r_{0}<0.2$ at $r=10 r_{0}$ and $k_{z} r_{0}<0.008$ at $r=25 r_{0}$, with relative amplitudes compared to those of the acoustic disturbances all the greater given that the Mach number is low. In Fig. 10 notable correlations also emerge approximately $10 r_{0} / c_{0}$ after the potential-core closing for $\delta z \simeq-40 r_{0}$, close to the line for a propagation at the speed of sound. Downstream-propagating noise components therefore appear to be detected, and to be emitted shortly after the potential-core closing, as will be discussed in what follows.

Apart from the axis-hydrodynamic correlations which may be identified around $\delta z \simeq-10 r_{0}$ for $M=0.6$, the results for $M=0.6,1.3$ and 2 in Figs. 9(d) and 9(e), 9(g) and 9(h), and 9(j) and $9(\mathrm{k})$ are very similar, suggesting the presence of a common noise generation mechanism. The peak correlations lie near the intersection of the dashed and solid lines, for negative separation distances. This supports that acoustic waves are emitted in the downstream direction around the time of potential-core closing. The correlations are negative for $u_{z}^{\prime}$ and positive for $|\vec{\omega}|^{\prime}$, which is due to the occurrence of velocity deficit and vorticity excess on the axis at $t \simeq t_{c}$ [25]. In absolute value, the correlation levels, which do not exceed 0.15 at $M=0.6$ and are greater than 0.25 for $M \geqslant 1.3$, increase with the jet velocity, as in the case for spatially developing jets [17]. Strong correlations are also encountered long after $t=t_{c}$ for the velocity fluctuations and before $t=t_{c}$ for the vorticity fluctuations, along trajectories revealing a velocity which is close to $0.65 u_{j}$ for $t \leqslant t_{c}$ and then decreases with time. This persistence of non-negligible correlations over a long period of time is related to the convection of the turbulent structures in the flow [18].

Finally, the normalized correlations between $p^{\prime}$ at $r=10 r_{0}$ and at $r=2.5 r_{0}$ are shown in Fig. 9 (right). For $M=0.3$, notable correlations are found but they do no occur along the solid line for a propagation of acoustic waves. They may be hydrodynamic-hydrodynamic correlations caused by the hydrodynamic components at $r=10 r_{0}$. For $M \geqslant 0.6$, on the contrary, significant correlations lie along elongated spots aligned with the solid line. The levels are stronger for a higher Mach number, and peak slightly after $t_{c}$. These features are similar to those exhibited by the axis-acoustic correlations, the correlations in the present case showing the links between the hydrodynamic pressure waves around the flows and the acoustic waves [9,72]. More specifically, in Figs. 9(f), 9(i), and 9(1), the correlations fork into two separate branches approximately for $\delta t \geqslant-10 r_{0} / c_{0}$. The branch on the left-hand side can be related to hydrodynamic-acoustic correlations between the sound 
(a)

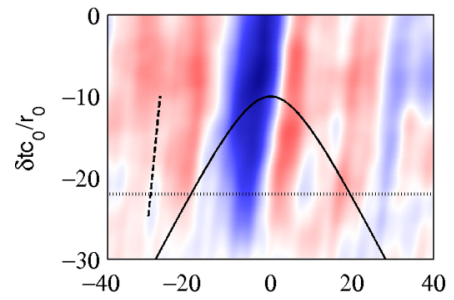

(d)

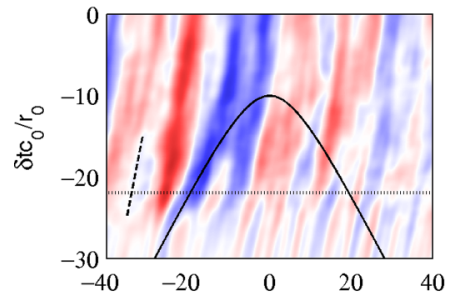

(g)

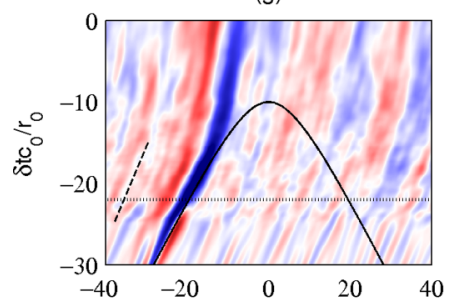

(j)

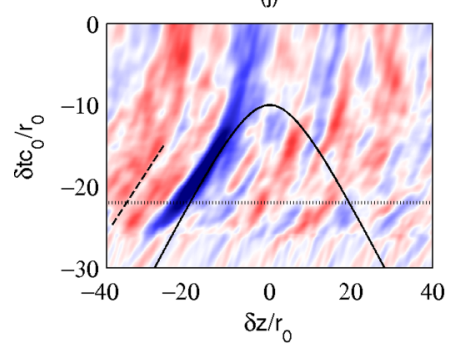

(b)

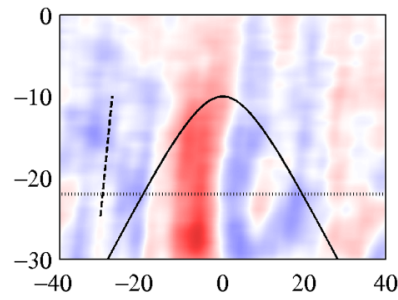

(e)

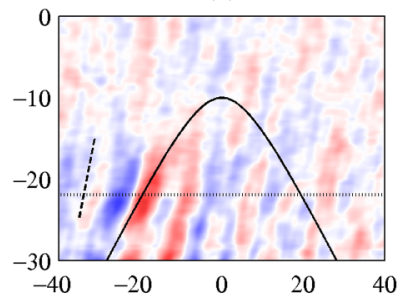

(h)

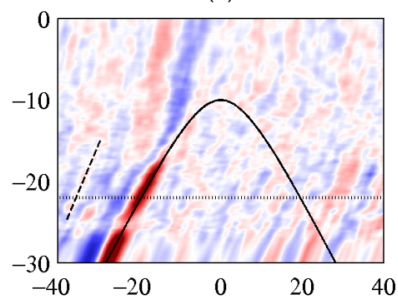

(k)

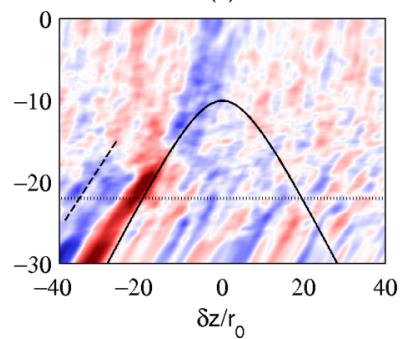

(c)

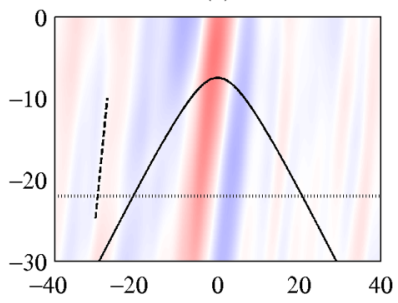

(f)

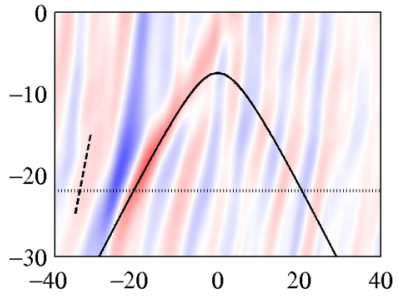

(i)

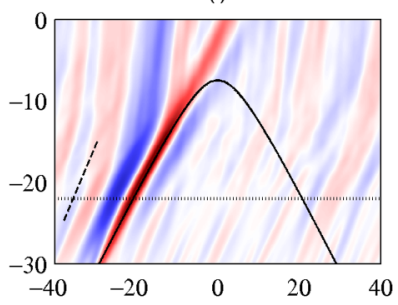

(l)

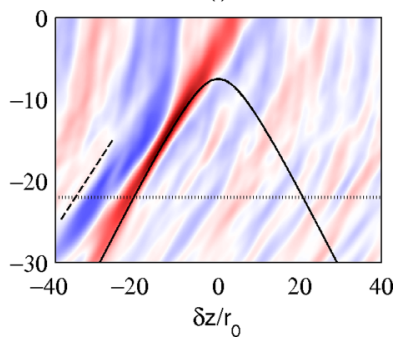

FIG. 9. Normalized correlations between $p^{\prime}\left(r_{\mathrm{ac}}, z, t_{\mathrm{ac}}\right)$ and (left) $u_{z}^{\prime}\left(r_{\mathrm{axis}}\right)$, (middle) $|\vec{\omega}|^{\prime}\left(r_{\mathrm{axis}}\right)$, (right) $p^{\prime}\left(r_{\text {hydro }}\right)$ at $z+\delta z$ and $t_{\mathrm{ac}}+\delta t$ for $n_{\theta}=0$, for $M=0.3,0.6,1.3$, and 2, from top to bottom; the color scales range between (left, middle) \pm 0.25 and (right) \pm 0.6 , from blue to red; $\delta t=t_{c}-t_{\mathrm{ac}} ;--$ velocity of $0.65 u_{j}$. propagation at $c_{0} ; \ldots$.

waves at $r_{\mathrm{ac}}$ and the hydrodynamic waves at $r_{\text {hydro }}$ convected at a decreasing velocity for $t \geqslant t_{c}$. That on the right-hand side corresponds to acoustic-acoustic correlations between the waves at $r_{\mathrm{ac}}$ and the lower part of the waves at $r_{\text {hydro }}$ propagating at $c_{0}$.

\section{E. Conditional averaging of the flow and pressure fields}

A conditional averaging method is employed in order to extract the noise generation mechanism related to the intrusion of turbulent structures in the potential core. The method consists in averaging samples selected using a trigger condition in a signal, and enables to isolate a specific phenomenon from the background noise. For jets, it has been used over the last years by Hileman et al. [73], 
(a)

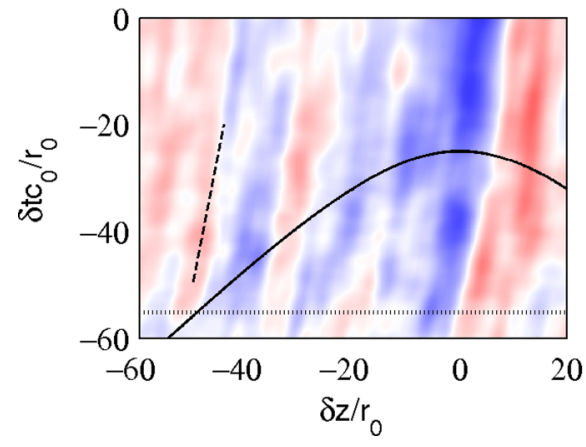

(b)

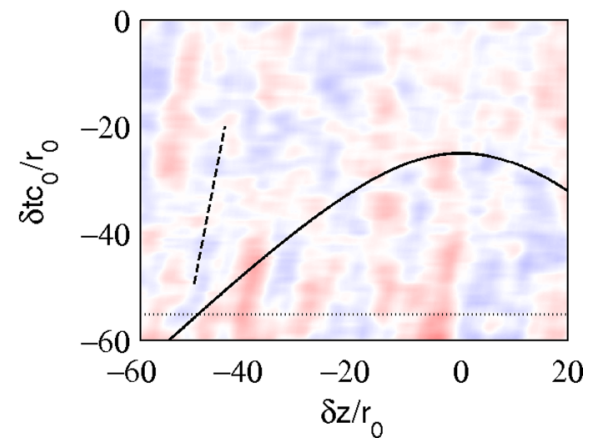

FIG. 10. Normalized correlations of $p^{\prime}\left(r=25 r_{0}, z, t=t_{c}+55 r_{0} / c_{0}\right)$ with (a) $u_{z}^{\prime}\left(r_{\text {axis }}\right)$ and (b) $|\vec{\omega}|^{\prime}\left(r_{\text {axis }}\right)$ at $z+\delta z$ and $t+\delta t$ for $M=0.3$. The color scale ranges between \pm 0.2 , from blue to red; _ propagation at $c_{0} ; \ldots . \delta t=t_{c}-t ;--$ velocity of $0.65 u_{j}$.

Kearney-Fisher et al. [74], and Camussi and Grizzi [75], for instance, and more recently by Schmidt and Schmid [41] and Pineau and Bogey [42] to study Mach-wave radiation in supersonic jets. In practice, the averaging procedure is very similar to that used for temporal jets at $M=0.9$ in Ref. [25], but it is here applied only to the axisymmetric mode of the flow and acoustic fields. The signal for the sample selection is the signal of axial velocity on the axis at the time of potential-core closing. The trigger events are identified by detecting the minimum values of velocity below a threshold of $0.7 u_{j}$. This threshold value is chosen arbitrarily, but it was verified in the aforesaid paper that the use of other thresholds, such as $0.60 u_{j}$ and $0.75 u_{j}$, only changes the results quantitatively. The trigger events separated by a distance of less than $7.5 r_{0}$ are discarded to avoid sample overlapping. This leads to a total number of samples equal to 40 for $M=0.3$, 46 for $M=0.6,61$ for $M=1.3$ and 34 for $M=2$, which is small but sufficient to obtain averaged fields clearly exhibiting coherent features. The flow and pressure fields are then recorded over windows centered around the position $z_{c}$ of the trigger events, and the resulting snapshots are ensemble averaged. This is carried out at time $t_{c}$, but also at previous and subsequent times using the same synchronization with the negative spikes of centerline velocity at $t_{c}$, yielding access to the time evolution of the generation and radiation of the axisymmetric sound components.

The conditional fields of velocity fluctuations in the flow and of pressure fluctuations outside at $t_{c}-8 r_{0} / u_{j}, t_{c}-4 r_{0} / u_{j}$ and $t_{c}$ are represented in Fig. 11. Those at $t_{c}+6 r_{0} / c_{0}, t_{c}+12 r_{0} / c_{0}$ and $t_{c}+18 r_{0} / c_{0}$ are shown in Fig. 12. The pressure fluctuations are multiplied by $M^{-2}$ for $t \leqslant t_{c}$ and by $M^{-3.75}$ for $t>t_{c}$, to get comparable levels for the hydrodynamic disturbances in the first case and for the acoustic ones in the second one. In the figures, velocity contours are drawn for the value of $75 \%$ of the mean centerline velocity to visualize the inner limit of the mixing layer. The time evolutions of the conditional fields of velocity and vorticity fluctuations in the flow and of pressure fluctuations outside can be seen in the movies available as Supplemental Material [62].

The results obtained before the potential-core closing in Fig. 11 share remarkable similarities regardless of the Mach number. In all jets, a spot of lower velocity and higher vorticity level relative to the background flow field develops at the inner edge of the mixing layer. It is located at $z \simeq$ $z_{c}-3 r_{0}$ in Figs. 11 (middle) and, by construction, $z=z_{c}$ in Figs. 11 (right). The axial length of this spot does not change much with the Mach number between $M=0.3$ and 1.3 but is longer for $M=2$. In the pressure field, a hydrodynamic wave centered on this velocity defect grows around the jet. With respect to the jet direction, its lobes are perpendicular for $M=0.3$ and $M=0.6$, but they are slightly inclined for $M=1.3$ and strongly inclined for $M=2$. In the latter case, they resemble Mach waves, which is not surprising given the supersonic motion of the flow structures [47].

The profiles of centerline velocity and vorticity fluctuations normalized by $u_{j}$ and $r_{0}$ and of pressure fluctuations at $r=2.5 r_{0}$ multiplied by $M^{-2}$, given by the conditional-averaging procedure 
(a)

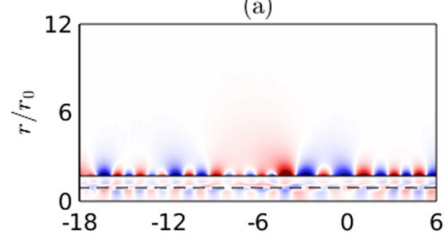

(d)

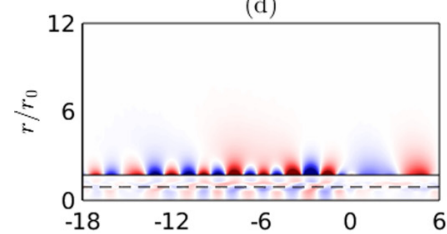

(g)

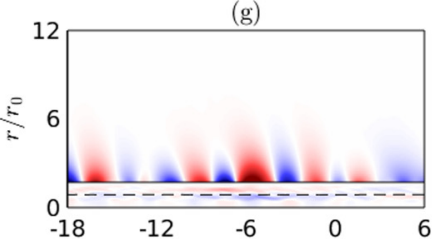

(j)

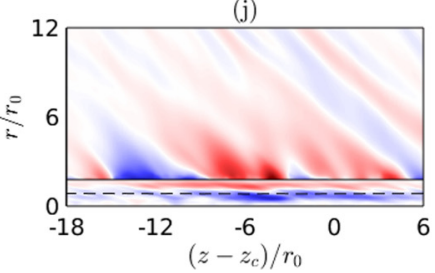

(b)

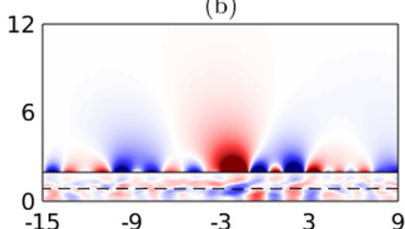

(e)

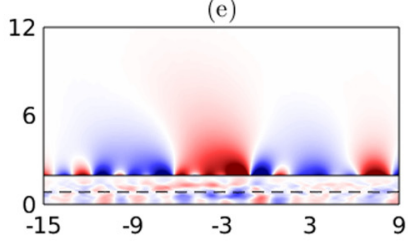

(h)

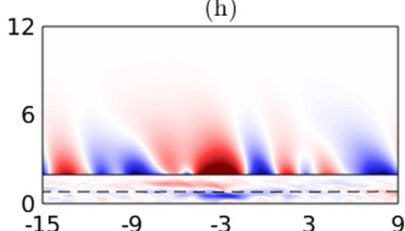

(k)

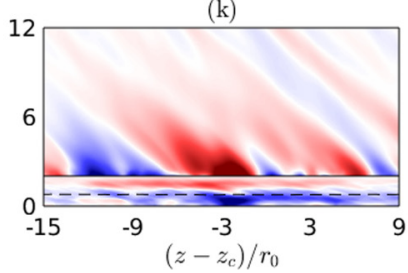

(c)

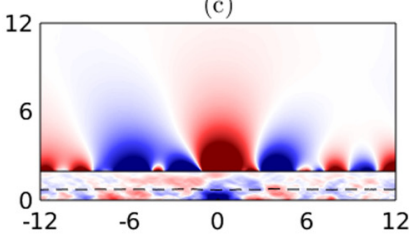

(f)

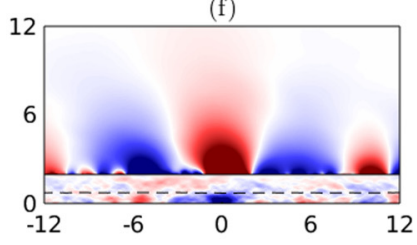

(i)

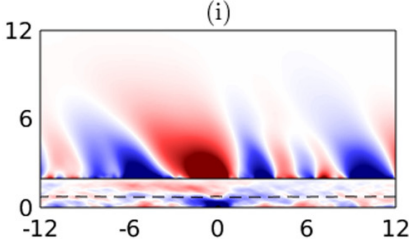

(1)

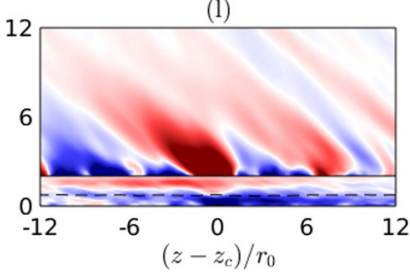

FIG. 11. Velocity fluctuations for $r \leqslant 1.75 r_{0}$ and pressure fluctuations otherwise, obtained using conditional averaging for $n_{\theta}=0$ at (left) $t_{c}-8 r_{0} / u_{j}$, (middle) $t_{c}-4 r_{0} / u_{j}$, and (right) $t_{c}$ for $M=0.3,0.6,1.3$, and 2, from top to bottom. The color scales range between $\pm 0.075 u_{j}$ and $\pm 0.0011 p_{0} M^{2}$, from blue to red; $---u_{z}=0.75\left\langle u_{z}(r=0)\right\rangle$. See also the movies available as Supplemental Material [62].

at $t=t_{c}$, are plotted in Figs. 13(a)-13(c). They do not depend appreciably on the Mach number in both shape and amplitude. In the velocity and vorticity profiles, negative and positive peaks of typical width of $0.6 r_{0}$ are found at $z=z_{c}$. They result from the arrival on the jet axis of the lowvelocity vortical spot developing in the inner side of the mixing layer. The hydrodynamic pressure waves induced by this spot appear in Fig. 13(c). They are dominated by a positive hump at $z=z_{c}$ for $M \leqslant 0.6$ and slightly more upstream for $M \geqslant 1.3$, and by a wavelength roughly of $8 r_{0}$.

After the potential-core closing, in Fig. 12, the spot of low velocity in the flow and its associated hydrodynamic pressure wave weaken, but persist over a long period of time and are still visible at $t_{c}+18 r_{0} / c_{0}$ for all jets. In the acoustic field, sound waves are emitted and propagate in the downstream direction. This is clearly the case for $M \geqslant 0.6$, but less obvious for $M=0.3$. The profiles of the pressure fluctuations at $r=10 r_{0}$ at $t_{c}+18 r_{0} / c_{0}$ are depicted in Fig. 13(d). For the four jets, a wavefront with an amplitude of $\left(p^{\prime} / p_{0}\right) \times M^{-3.75} \simeq 5 \times 10^{-4}$ is observed. It is however located farther upstream as the Mach number decreases. This is particularly true for $M=0.3$, which further supports the contention of previous section that this jet radiates noise components in the downstream direction as the other jets, but a later time. Note that correlations computed between the conditional fields of $p^{\prime}$ at $r=10 r_{0}$ and the centerline velocity fluctuations for $M=0.6,1.3$ and 2 are displayed in Appendix B. They are in good qualitative agreement with the 
(a)

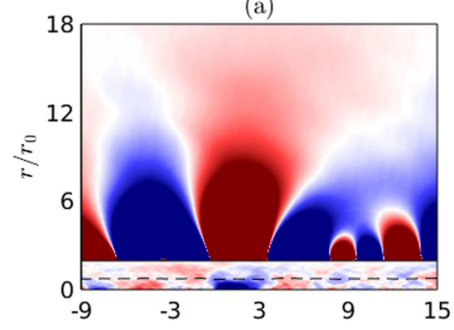

(d)

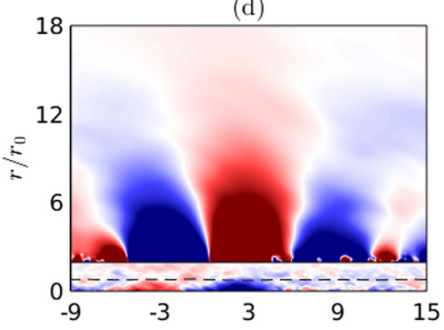

(g)

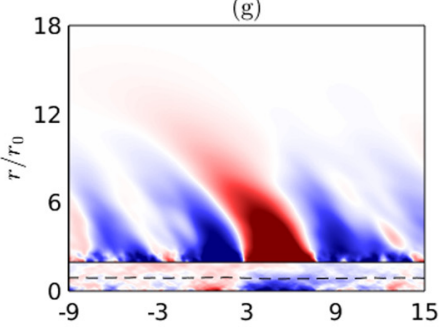

(j)

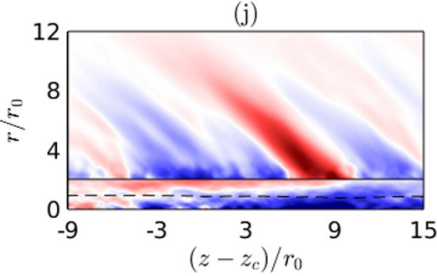

(b)

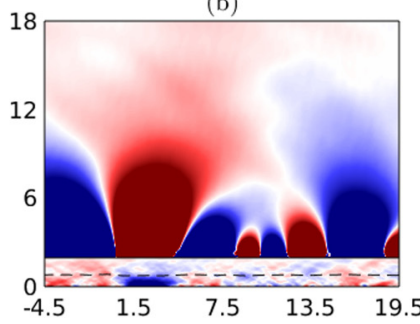

(e)

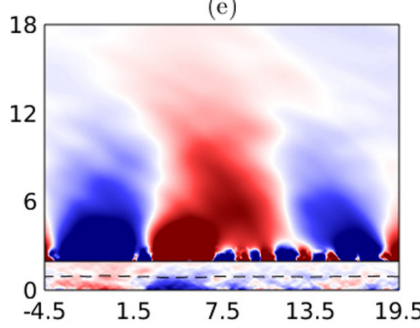

(h)

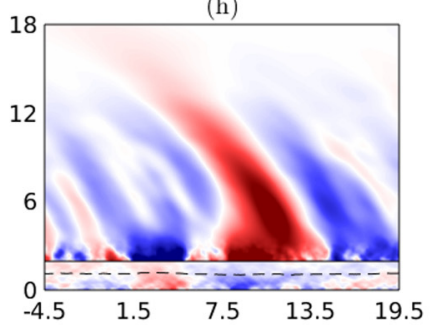

(k)

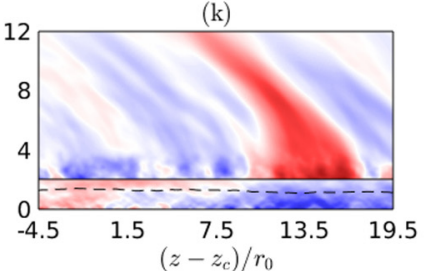

(c)

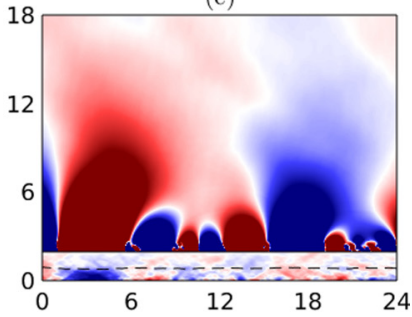

(f)

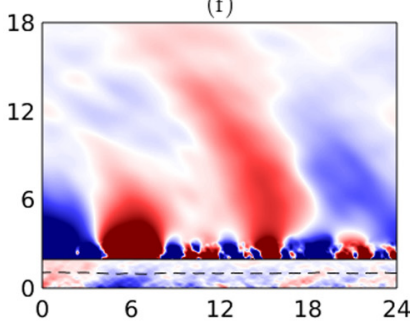

(i)

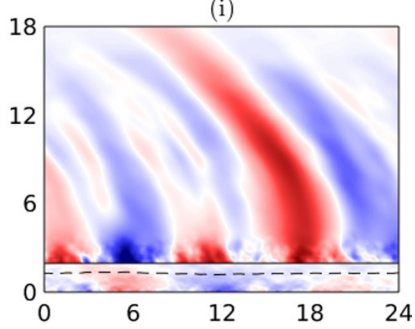

(1)

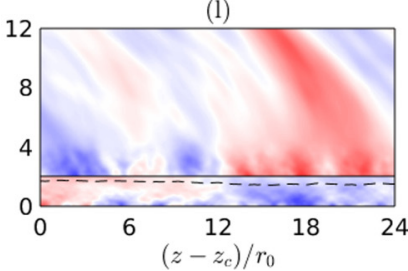

FIG. 12. Velocity fluctuations for $r \leqslant 1.75 r_{0}$ and pressure fluctuations otherwise, obtained using conditional averaging for $n_{\theta}=0$ at (left) $t_{c}+6 r_{0} / c_{0}$, (middle) $t_{c}+12 r_{0} / c_{0}$ and (right) $t_{c}+18 r_{0} / c_{0}$ for $M=0.3$, $0.6,1.3$, and 2 , from top to bottom. The color scales range between $\pm 0.075 u_{j}$ and $\pm 0.0011 p_{0} M^{3.75} \mathrm{~Pa}$, from blue to red; $---u_{z}=0.75\left\langle u_{z}(r=0)\right\rangle$. See also the movies available as Supplemental Material [62].

correlations in Sec. III D, indicating that the noise generation mechanism extracted using conditional averaging corresponds to the source revealed using the latter correlations.

The time variations of the minimum values of velocity in the flow and of the maximum values of pressure at $r=2.5 r_{0}$ are shown over $t_{c}-8 r_{0} / u_{j} \leqslant t \leqslant t_{c}+8 r_{0} / u_{j}$ in Figs. 14(a) and 14(b). For all jets, in absolute value, they first rapidly grow, peak and then gradually decay. The peak is located at $t_{c}$ for $u_{z}^{\prime}$ and at $t_{c}$ or slightly later for $p^{\prime}$. In addition, the peak is narrower, i.e., the growing-and-decaying process happens over a shorter period of time, for $u_{z}^{\prime}$ than for $p^{\prime}$. The axial positions of the minimum values of velocity are illustrated in Fig. 14(c) to gain information on the convection velocity of the spot of velocity deficit. In all cases, this velocity is approximately of $0.65 u_{j}$ at $t=t_{c}$, and then decreases. Before $t_{c}$, it seems to be slightly lower than $0.65 u_{j}$ for $M=0.3$, 0.6 and 2 , and close to this value for $M=1.3$. This is confirmed by the profiles of Fig. 14(d) 

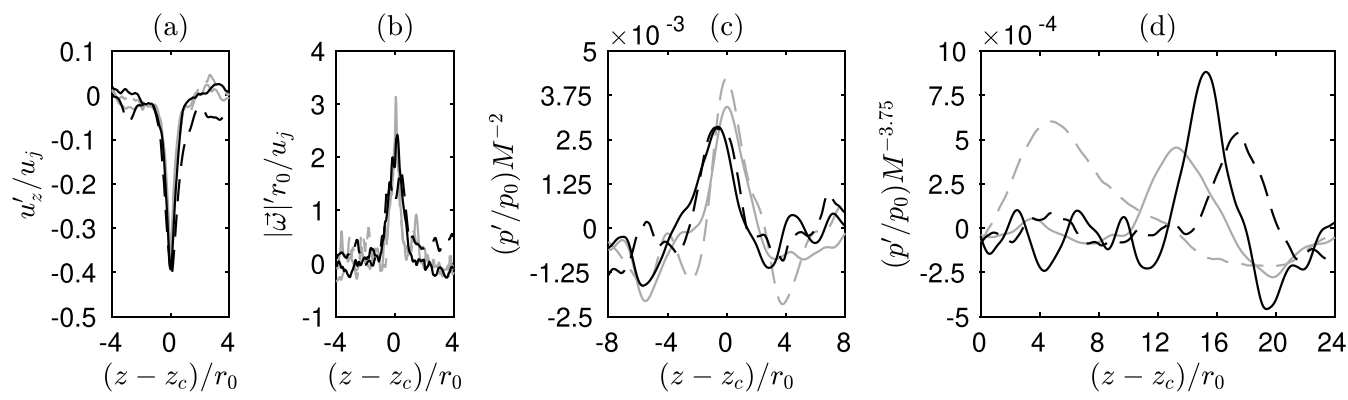

FIG. 13. Velocity, vorticity and pressure fluctuations obtained using conditional averaging for $n_{\theta}=0$, (a) $u_{z}^{\prime}\left(r_{\text {axis }}, t_{c}\right) / u_{j}$ and (b) $|\vec{\omega}|^{\prime}\left(r_{\text {axis }}, t_{c}\right) r_{0} / u_{j}$, (c) $\left(p^{\prime}\left(r_{\text {hydro }}, t_{c}\right) / p_{0}\right) \times M^{-2}$ and (d) $\left(p^{\prime}\left(r_{\text {ac }}, t_{c}+18 r_{0} / c_{0}\right) / p_{0}\right) \times$ $M^{-3.75}:--M=0.3$, $M=0.6$, $M=1.3,--M=2$.

showing the time derivatives of the positions depicted in Fig. 14(c), except for $M=2$ because of strong oscillations. Therefore, as previously pointed out for jets at $M=0.9$ in Ref. [25], no strong acceleration is noticed when the potential core closes, which is in contrast with the convection velocities acquired from the correlation functions of centerline velocity fluctuations of Fig. 5(b).

The variations of the maximum values of $p^{\prime}$ at $r=10 r_{0}$ and of their axial positions between $t_{c}$ and $t_{c}+24 r_{0} / u_{j}$ are provided in Figs. 15(a) and 15(b). For $M=0.3$, significant levels are obtained in Fig. 15(a) for $t \leqslant t_{c}+12 r_{0} / u_{j}$ at positions remaining close to $z=0$. They might be due to the hydrodynamic disturbances mentioned in the previous section. Later, the pressure levels exhibit a second hump for $t \simeq t_{c}+19 r_{0} / u_{j}$, which is most likely of acoustic nature. For $M=0.6,1.3$, and 2 , the pressure levels increase and reach peak values around $t_{c}+16 r_{0} / u_{j}$, as expected according to the rms profiles of Fig. 7. Apart for $M=0.6$ for $t \leqslant t_{c}+8 r_{0} / u_{j}$, certainly also due to the presence of hydrodynamic disturbances, the axial positions of the pressure extrema increase as the acoustic waves propagate. In all cases, in addition, they indicate a propagation velocity tending toward the speed of sound as the direction of noise radiation is closer to the axial direction. Moreover, for $t \geqslant t_{c}+12 r_{0} / u_{j}$, the distance $z-z_{c}$ is shorter at a lower Mach number, which is consistent with Fig. 13(d).

The radiation angles computed from the positions of the pressure peaks at $r=10 r_{0}$ for $M=$ $0.6,1.3$, and 2 are presented in Fig. 15(c) between $t_{c}+10 r_{0} / u_{j}$ and $t_{c}+22 r_{0} / u_{j}$. For $t \geqslant t_{c}+$ $15 r_{0} / u_{j}$, similar results are found with angles decreasing from $\phi \simeq 40$ down to $\phi \simeq 30^{\circ}$ relative to the flow direction. These angles are lower than those in Fig. 7(c) obtained from correlations of the full pressure fluctuations. This may be due to the fact that in the conditional pressure fields the noise components generated by the potential-core closing are isolated from other components by

(a)

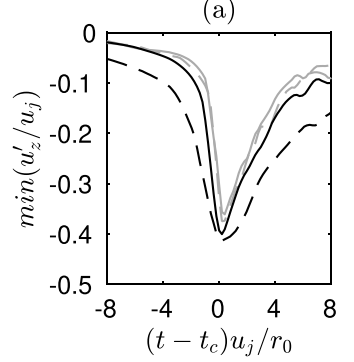

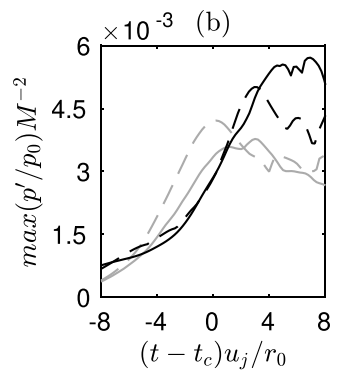
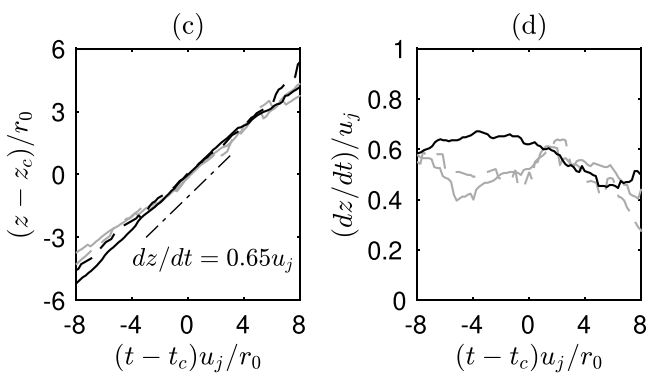

FIG. 14. Time variations of (a) the minimum of $u_{z}^{\prime} / u_{j}$ over $0 \leqslant r \leqslant r_{0}$, (b) the maximum of $p^{\prime} / p_{0}$ at $r_{\text {hydro }}$, (c) the axial position of the minimum of $u_{z}^{\prime}$ and (d) its time derivative, obtained using conditional averaging for $n_{\theta}=0:--M=0.3$, $M=0.6$, $M=1.3, \ldots--M=2 ; \_-\ldots$ velocity of $0.65 u_{j}$. 

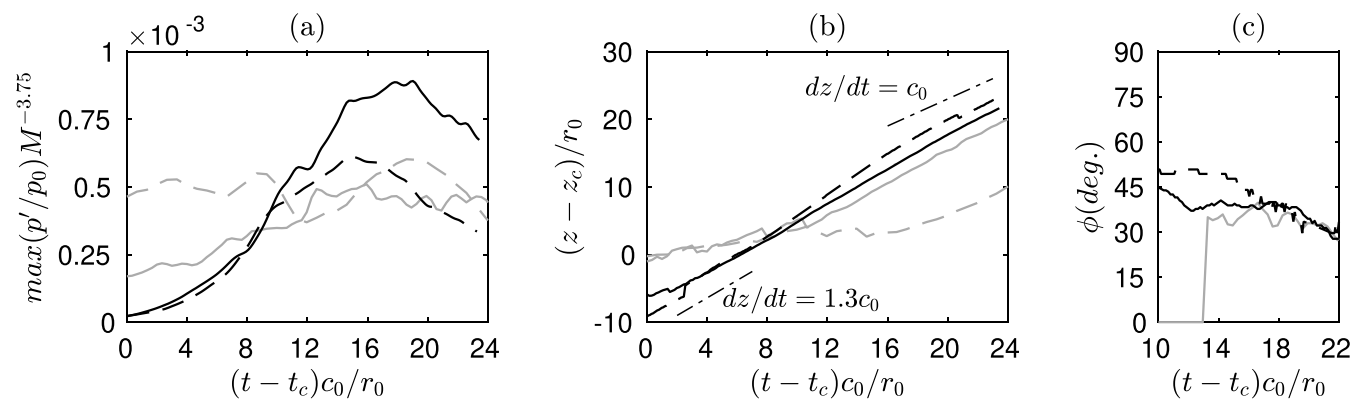

FIG. 15. Time variations of (a) the maximum of $p^{\prime} / p_{0}$ at $r_{\mathrm{ac}}$, (b) their axial positions and (c) the corresponding radiation angles relative to the jet direction, obtained using conditional averaging for $n_{\theta}=0$ : $=-M=0.3$ $M=0.6$, $M=1.3, \ldots-{ }_{-} M=2 ; \_-$. velocity of $c_{0}$ and $1.3 c_{0}$.

the averaging procedure. As in Fig. 7(c), however, the angle for $M=2$ remains close to the angle of $54^{\circ}$ previously associated with Mach-wave radiation.

Finally, an attempt is made to quantitatively relate the flow features at potential-core closing and the emitted sound waves. For that, conditional averaging is performed as above but with different trigger conditions for $M=0.6$ and 1.3. For $M=0.6$, the DNS fields are synchronized with the minimum values of centerline velocity $u_{z}^{\prime}$ at $t_{c}$ below a threshold of $0.6 u_{j}$ in a first case, and between $0.6 u_{j}$ and $0.75 u_{j}$ in a second one, yielding conditional fields computed from 20 and 44 samples, respectively. For $M=1.3$, in the same way, the trigger conditions are $u_{z}^{\prime}\left(r=0, t_{c}\right)<0.55 u_{j}$ and $0.55 u_{j} \leqslant u_{z}^{\prime}\left(r=0, t_{c}\right) \leqslant 0.75 u_{j}$ to obtain 21 and 52 samples in the two cases. The conditional fields of velocity and pressure thus determined for $M=1.3$ are illustrated in Fig. 16 at $t_{c}, t_{c}+9 r_{0} / c_{0}$ and $t_{c}+18 r_{0} / c_{0}$. Overall, the results are very similar using the two trigger conditions, but the amplitudes of the fluctuations differ and are higher in Figs. 16 (bottom) using $u_{z}^{\prime}\left(r=0, t_{c}\right)<0.55 u_{j}$ for the sample selection than in Figs. 16 (top).

Some properties of the conditional fields are brought together. The maximum values of vorticity norm in the flow for $M=0.6$ and 1.3 are presented in Fig. 17(a) as a function of the absolute value of the minimum of velocity. They increase monotonically, indicating that the more marked the velocity deficit during the potential-core closing, the stronger the vorticity excess. Inversely, the

(a)

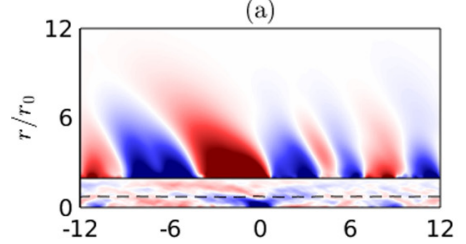

(d)

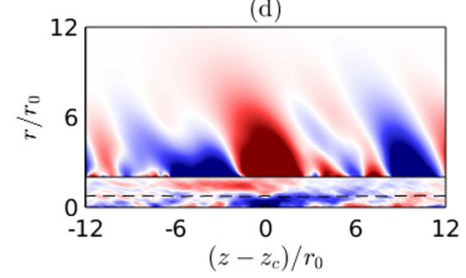

(b)

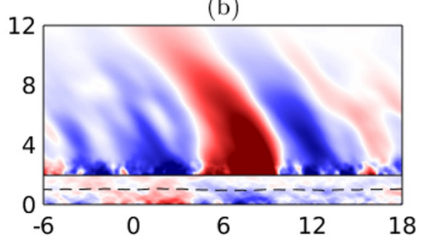

(e)

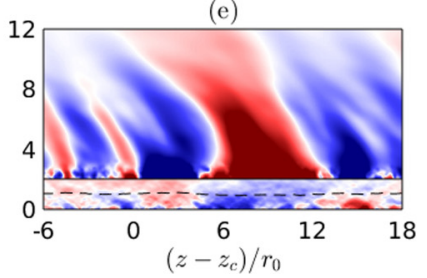

(c)

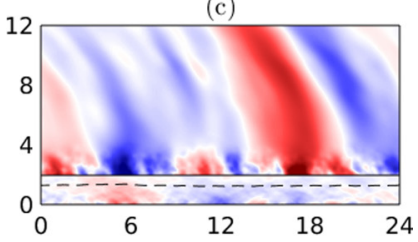

(f)

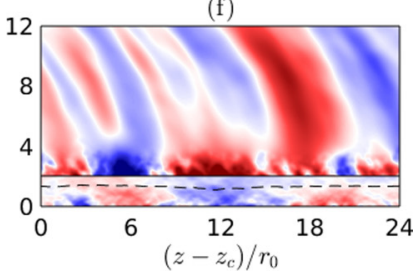

FIG. 16. Velocity fluctuations for $r \leqslant 1.75 r_{0}$ and pressure fluctuations otherwise, obtained for $M=1.3$ at (left) $t_{c}$, (middle) $t_{c}+9 r_{0} / c_{0}$ and (right) $t_{c}+18 r_{0} / c_{0}$ for $n_{\theta}=0$, using the trigger conditions (top) $0.55 u_{j} \leqslant$ $u_{z}^{\prime}\left(r=0, t_{c}\right) \leqslant 0.75 u_{j}$ and (bottom) $u_{z}^{\prime}\left(r=0, t_{c}\right)<0.55 u_{j}$. The color scales range between $\pm 0.075 u_{j}$ and $\pm 0.0011 p_{0} M^{3.75} \mathrm{~Pa}$, from blue to red; $--u_{z}=0.75\left\langle u_{z}(r=0)\right\rangle$. 
(a)

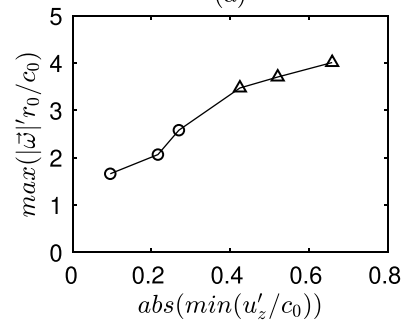

(b)

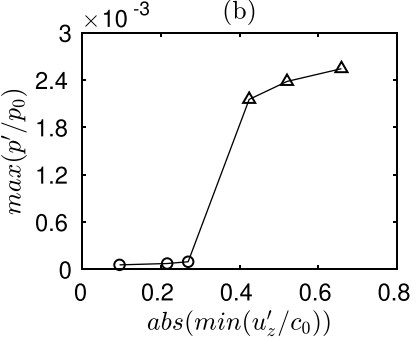

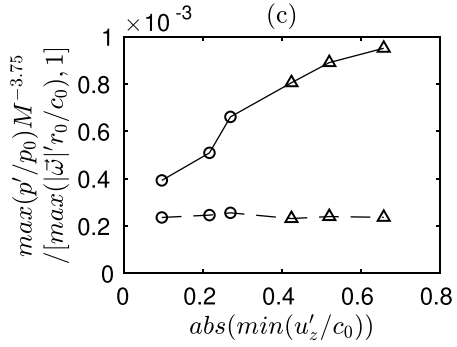

FIG. 17. Conditional averages for $\circ M=0.6$ and $\triangle M=1.3$ for $n_{\theta}=0$ : (a) maximum of $|\vec{\omega}|^{\prime} r_{0} / c_{0}$ over $0 \leqslant r \leqslant r_{0}$, (b) maximum of $p^{\prime} / p_{0}$ at $r_{\text {ac }}$ and (c) maximum of $p^{\prime} / p_{0}$ at $r_{\text {ac }}$ multiplied by $M^{-3.75}$ and $---M^{-3.75} / \max \left(|\vec{\omega}|^{\prime} r_{0} / c_{0}\right)$, as a function of the minimum of $u_{z}^{\prime} / c_{0}$ over $0 \leqslant r \leqslant r_{0}$.

intense structures entering in the potential core lead to a deeper drop of the centerline velocity than weaker ones. Looking at the sound fields, the maximum values of pressure fluctuations at $r=10 r_{0}$ are plotted in Fig. 17(b). Two well-distinct steps, one for $M=0.6$ and another for $M=1.3$, are observed, on which the levels grow with the velocity deficit. The pressure peaks are multiplied by $M^{-3.75}$ and $M^{-3.75} / \max \left(|\vec{\omega}|^{\prime} r_{0} / c_{0}\right)$ in Fig. 17(c). The values obtained increase in the first case, whereas they do not vary much in the second one. Therefore, the amplitude of the acoustic waves appears to be a function of the Mach number, as expected, but also of the strength of the vortical structures closing the potential core or, given the results of Fig. 17(a), of the velocity deficit. The latter links seem to be linear, but it is difficult to conclude this definitively because of the limited number of samples in the conditional averages.

\section{CONCLUSION}

In this paper, the generation of noise components by the potential-core closing of temporally developing isothermal round jets at a Reynolds number of 3125 and Mach numbers of 0.3, 0.6, 1.3 , and 2 is investigated using direct numerical simulation. For that purpose, the main properties of the flow and pressure fields are compared, flow-noise cross-correlations are shown to identify source regions in the flow, and a conditional-averaging procedure is employed to identify the generation mechanisms. At a higher Mach number, the jet develops more slowly due to the weaker amplification rates of the shear-layer instability waves, leading to a later time of closing of the potential core. At that time, however, the spectra of velocity in the flow and of hydrodynamic pressure in its immediate vicinity are similar to each other when plotted as a function of the axial wave number $k_{z}$ and multiplied by $M^{-2}$ and $M^{-4}$, respectively. In the acoustic field, axisymmetric sound waves are emitted in the downstream direction. For $M \leqslant 1.3$, their corresponding spectra scale as $k_{z} M^{-1}$ in frequency and as $M^{7.5}$ in level. For $M \geqslant 1.3$, a frequency scaling with $k_{z}$, as well as a lower power-law exponent for the levels as expected for supersonic jets, seem to apply. For $M=2$, in addition, the downstream sound waves look like Mach waves, whereas they do not in the other cases. Despite this, they appear to be generated by a common mechanism in the four cases with Mach numbers from 0.3 to 2 .

Some characteristics of that mechanism are revealed by the conditional-averaged fields synchronized with the peaks of velocity deficit on the jet axis at the time of potential-core closing. The generation mechanism occurs over a long period of time of the order of $10 r_{0} / u_{j}$, and predominantly emits noise around the potential-core closing. As previously in Ref. [25] for temporally developing jets at $M=0.9$, it is shown to be related to the development in the inner part of the jet mixing layer, the rapid intrusion on the jet axis, and then the weakening, of a structure of lower velocity and higher vorticity level with respect to the background flow field, and to the associated growth-and-decay process of a hydrodynamic pressure wave just outside of the jet. This mechanism is observed over the wide range of Mach numbers considered, in particular at both $M=0.3$ and $M=2$, which is 
rather surprising given the difference in Mach number. Moreover, using different trigger conditions for the conditional averaging, the amplitude of the waves generated at the potential-core closing is found to depend on the peak values of the velocity deficit and vorticity excess in the flow at that time, possibly in a linear way. The present results provide original information on the axisymmetric downstream-propagating jet noise component usually attributed to the jet large-scale structures. It is hoped that, combined with results obtained for spatially developing jets at various Mach and Reynolds numbers, they will help us to get a better understanding of the source responsible for this component.

\section{ACKNOWLEDGMENTS}

The present work was granted access to the High-Performance-Computing resources of FLMSN (Fédération Lyonnaise de Modélisation et Sciences Numériques), partner of EQUIPEX EQUIP@MESO, and of IDRIS (Institut du Développement et des Ressources en Informatique Scientifique) under the allocation 2017-2a0204 made by GENCI (Grand Equipement National de Calcul Intensif). It was performed within the framework of the LABEX CeLyA(ANR-10LABX-0060) of Université de Lyon, within the program Investissements d'Avenir (ANR-16-IDEX0005) operated by the French National Research Agency (ANR). Finally, the authors gratefully acknowledge Roberto Sabatini for performing linear stability analyses from the jet flow profiles.

\section{APPENDIX A}

In this first Appendix, some results obtained by the linear stability analysis documented in Sec. II D between $t=t_{c}-15 r_{0} / u_{j}$ and $t_{c}$ for the axisymmetric mode are presented. The axial wave numbers and the growth rates of the most unstable waves, normalized by the momentum thickness, are shown in Figs. 18(a) and 18(b). Both are lower at higher Mach numbers, as expected [60]. For $M=0.3$, in particular, their values at $t=t_{c}-15 r_{0} / u_{j}$ are close to those obtained in the temporal linear stability analysis of Michalke [59] for an incompressible two-dimensional mixing layer, namely 0.22 and 0.047 , respectively. As time passes, that is, as the mixing-layer thickness increases and tends to the jet radius, the peak Strouhal number slightly decreases in all cases. Meanwhile, the instability growth rates collapse, resulting in no unstable wave number around the time of potential-core closing. However, the higher the Mach number, the earlier the time when all disturbances are damped. This arises at $t_{c}-1.5 r_{0} / u_{j}$ for $M=0.3, t_{c}-1.9 r_{0} / u_{j}$ for $M=0.6$, $t_{c}-2.7 r_{0} / u_{j}$ for $M=0.9$ and 1.3 , and $t_{c}-13 r_{0} / u_{j}$ for $M=2$.

The axial wave numbers and growth rates of the most unstable waves are replotted in Figs. 19(a) and 19(b) using a scaling with the jet radius, i.e. a length scale which does not evolve with time. Both

(a)

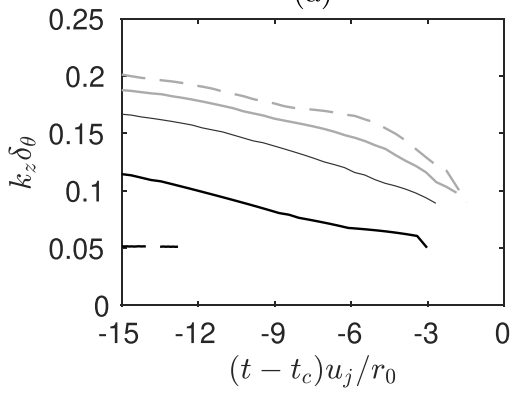

(b)

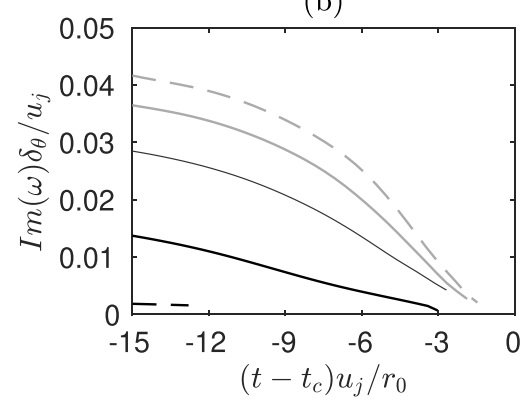

FIG. 18. Time variations of (a) the axial wave number and (b) the growth rate of the most unstable wave for $n_{\theta}=0$, normalized by $\delta_{\theta}:---M=0.3$, $M=0.6$, $M=0.9$, $M=1.3,-$ $M=2$. 
(a)

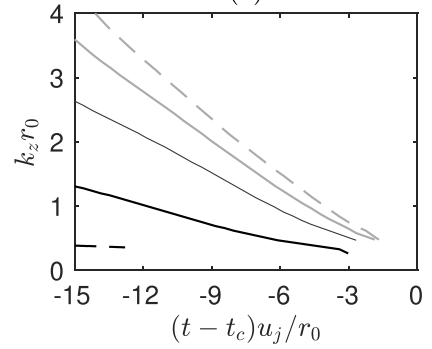

(b)

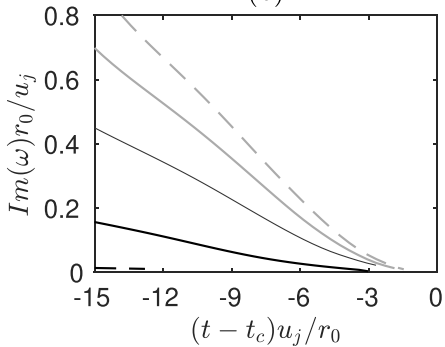

(c)

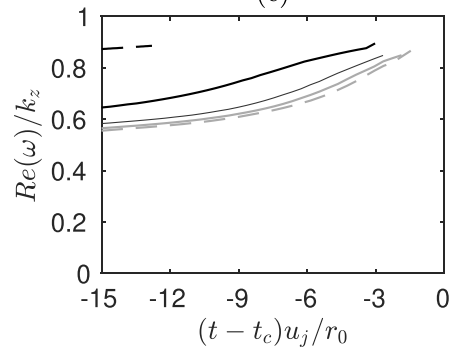

FIG. 19. Time variations of (a) the axial wave number, (b) the growth rate and (c) the phase velocity of the most unstable wave for $n_{\theta}=0$, normalized by $r_{0}$ : $--M=0.3$, $M=0.6$, $M=0.9$, $M=1.3,--M=2$.

sharply decrease as the shear layer spreads. Furthermore, the last unstable wave numbers are equal to $k_{z} r_{0} \simeq 0.45$ for $M=0.3,0.6$, and 0.9 , and $k_{z} r_{0} \simeq 0.35$ for $M=1.3$ and 2 . For completeness, the phase velocities of the most unstable waves are given in Fig. 19(c). For $M \leqslant 1.3$, they are close to $0.6 u_{j}$ at $t=t_{c}-15 r_{0} / u_{j}$ and then progressively increase to reach peak values around $0.8 u_{j}$ before the potential-core closing. For $M=2$, such values are found well before, following the shift in time observed in Figs. 19(a) and 19(b) between this case and the others.

\section{APPENDIX B}

In this second Appendix, correlations are computed between the conditional fields of pressure fluctuations at $\left(r=10 r_{0}, z\right)$ at $t=t_{c}+15 r_{0} / c_{0}$ and velocity fluctuations at $(r=0, z+\delta z)$ at $t+$ $\delta t$ for $M=0.6,1.3$ and 2. The maps of normalized correlations are shown in Figs. 20 (top) as

(a)

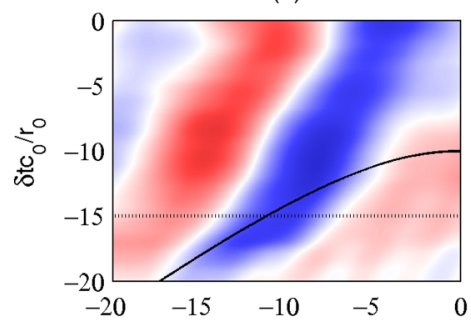

(d)

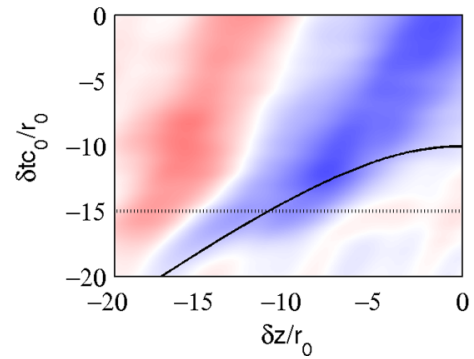

(b)

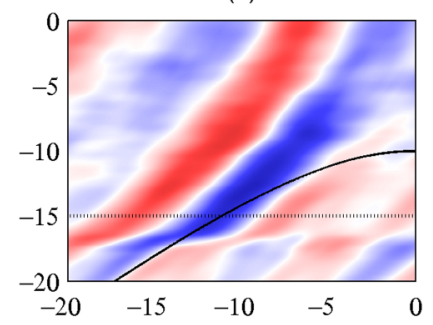

(e)

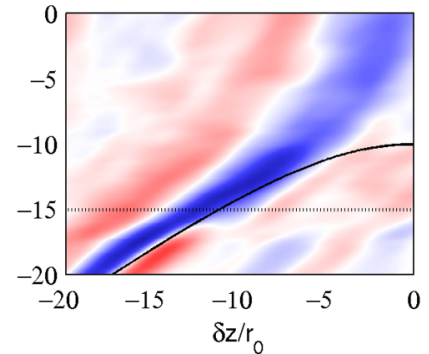

(c)

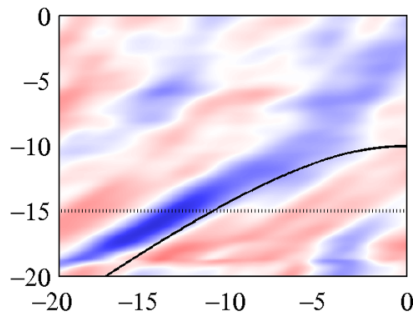

(f)

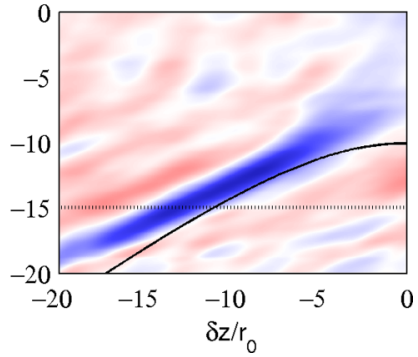

FIG. 20. Normalized correlations between $p^{\prime}\left(r_{\mathrm{ac}}, z, t=t_{c}+15 r_{0} / c_{0}\right)$ and $u_{z}^{\prime}\left(r_{\mathrm{axis}}, z+\delta z, t+\delta t\right)$ from (top) the conditionally averaged and (bottom) the full fields for $n_{\theta}=0$ : (left) $M=0.6$, (middle) $M=1.3$, (right) $M=2$. The color scales range between (top) \pm 1 and (bottom) \pm 0.45 , from blue to red; propagation at $c_{0} ; \ldots . \delta t=t_{c}-t$. 
a function of $\delta z / r_{0}$ and $\delta t c_{0} / r_{0}$, and are compared with those of Figs. 20 (bottom), determined from the full fields of pressure and velocity. The correlation levels are higher in Figs. 20 (top) as expected given the selection of the strongest events using the conditional-averaging procedure, but the correlation patterns are very similar in the two cases. The noise generation mechanism extracted using conditional averaging in Sec. III E therefore appears to be responsible for the flow-noise correlations of Sec. III D.

[1] M. J. Lighthill, On sound generated aerodynamically I. General theory, Proc. Roy. Soc. A 211, 564 (1952).

[2] H. S. Ribner, The generation of sound by turbulent jets, Adv. Appl. Mech. 8, 103 (1964).

[3] A. Powell, Theory of vortex sound, J. Acoust. Soc. Am. 36, 177 (1964).

[4] E. Mollo-Christensen, Jet noise and shear flow instability seen from an experimenter's viewpoint, J. Appl. Mech 34, 1 (1967).

[5] R. A. Petersen, R. E. Kaplan, and J. Laufer, Ordered structures and jet noise, Tech. Rep. 134733, NASA CR (1974).

[6] C. K. W. Tam and L. Auriault, Jet mixing noise from fine-scale turbulence, AIAA J. 37, 145 (1999).

[7] J. E. Ffowcs Williams and A. J. Kempton, The noise from the large-scale structure of a jet, J. Fluid Mech. 84, 673 (1978).

[8] C. K. W. Tam, Supersonic jet noise, Annu. Rev. Fluid Mech. 27, 17 (1995).

[9] C. K. W. Tam, K. Viswanathan, K. K. Ahuja, and J. Panda, The sources of jet noise: Experimental evidence, J. Fluid Mech. 615, 253 (2008).

[10] D. Juvé, M. Sunyach, and G. Comte-Bellot, Filtered azimuthal correlations in the acoustic far field of a subsonic jet, AIAA J. 17, 112 (1979).

[11] A. V. G. Cavalieri, P. Jordan, T. Colonius, and Y. Gervais, Axisymmetric superdirectivity in subsonic jets, J. Fluid Mech. 704, 388 (2012).

[12] C. K. W. Tam, Jet noise: Since 1952, Theor. Comput. Fluid Dyn. 10, 393 (1998).

[13] W. T. Chu and R. E. Kaplan, Use of a spherical concave reflector for jet-noise-source distribution diagnosis, J. Acoust. Soc. Am. 59, 1268 (1976).

[14] M. J. Fisher, M. Harper-Bourne, and S. A. L. Glegg, Jet engine noise source location: The polar correlation technique, J. Sound Vib. 51, 23 (1977).

[15] S. S. Lee and J. Bridges, Phased-array measurements of single flow hot jets, NASA/TM 2005-213826 (2005).

[16] M. Schaffar, Direct measurements of the correlation between axial in-jet velocity fluctuations and far field noise near the axis of a cold jet, J. Sound Vib. 64, 73 (1979).

[17] J. Panda, R. G. Seasholtz, and K. A. Elam, Investigation of noise sources in high-speed jets via correlation measurements, J. Fluid Mech. 537, 349 (2005).

[18] C. Bogey and C. Bailly, An analysis of the correlations between the turbulent flow and the sound pressure field of subsonic jets, J. Fluid Mech. 583, 71 (2007).

[19] J. L. Stromberg, D. K. McLaughlin, and T. R. Troutt, Flow field and acoustic properties of a Mach number 0.9 jet at a low Reynolds number, J. Sound. Vib. 72, 159 (1980).

[20] J. B. Freund, Noise sources in a low-Reynolds-number turbulent jet at Mach 0.9, J. Fluid Mech. 438, 277 (2001).

[21] E. Mollo-Christensen, M. A. Kolpin, and J. R. Martucelli, Experiments on jet flows and jet noise far-field spectra and directivity patterns, J. Fluid Mech. 18, 285 (1964).

[22] C. Bogey, S. Barré, V. Fleury, C. Bailly, and D. Juvé, Experimental study of the spectral properties of near-field and far-field jet noise, Int. J. Aeroacoust. 6, 73 (2007).

[23] K. B. M. Q. Zaman and J. C. Yu, Power spectral density of subsonic jet noise, J. Sound Vib. 98, 519 (1985).

[24] K. Ahuja and K. Bushell, An experimental study of subsonic jet noise and comparison with theory, J. Sound Vib. 30, 317 (1973). 
[25] C. Bogey, On noise generation in low Reynolds number temporal round jets at a Mach number of 0.9, J. Fluid Mech. 859, 1022 (2019).

[26] P. Comte, M. Lesieur, and E. Lamballais, Large- and small-scale stirring of vorticity and a passive scalar in a 3-D temporal mixing layer, Phys. Fluids A 4, 2761 (1992).

[27] M. M. Rogers and R. D. Moser, Direct simulation of a self-similar turbulent mixing layer, Phys. Fluids 6, 903 (1994).

[28] A. W. Vreman, N. D. Sandham, and K. H. Luo, Compressible mixing layer growth rate and turbulence characteristics, J. Fluid Mech. 320, 235 (1996).

[29] J. B. Freund, S. K. Lele, and P. Moin, Compressibility effects in a turbulent annular mixing layer. Part 1. Turbulence and growth rate, J. Fluid Mech. 421, 229 (2000).

[30] V. Fortuné, E. Lamballais, and Y. Gervais, Noise radiated by a non-isothermal, temporal mixing layer. Part I: Direct computation and prediction using compressible DNS, Theor. Comput. Fluid Dyn. 18, 61 (2004).

[31] R. R. Kleimann and J. B. Freund, The sound from mixing layers simulated with different ranges of turbulence scales, Phys. Fluids 20, 101503 (2008).

[32] D. A. Buchta and J. B. Freund, The near-field pressure radiated by planar high-speed free-shear-flow turbulence, J. Fluid Mech. 832, 383 (2017).

[33] D. A. Buchta and J. B. Freund, Intense sound radiation by high-speed flow: Turbulence structure, gas properties, and near-field gas dynamics, Phys. Rev. Fluids 4, 044605 (2019).

[34] D. A. Buchta, G. Shallcross, and J. Capecelatro, Sound and turbulence modulation by particles in highspeed shear flows, J. Fluid Mech. 875, 254 (2019).

[35] T. Suzuki, Coherent noise sources of a subsonic round jet investigated using hydrodynamic and acoustic phased-microphone arrays, J. Fluid Mech. 730, 659 (2013).

[36] A. V. G. Cavalieri, D. Rodrìguez, P. Jordan, T. Colonius, and Y. Gervais, Wavepackets in the velocity field of turbulent jets, J. Fluid Mech. 730, 559 (2013).

[37] D. Papamoschou, Wavepacket modeling of the jet noise source, Int. J. Aeroacoust. 17, 52 (2018).

[38] N. D. Sandham, C. L. Morfey, and Z. W. Hu, Nonlinear mechanisms of sound generation in a perturbed parallel jet flow, J. Fluid Mech. 565, 1 (2006).

[39] J. E. Ffowcs Williams, The noise from turbulence convected at high speed, Phil. Trans. R. Soc. Lond. A 255, 469 (1963).

[40] J. Ryu, S. K. Lele, and K. Viswanathan, Study of supersonic wave components in high-speed turbulent jets using an LES database, J. Sound Vib. 333, 6900 (2014).

[41] O. T. Schmidt and P. J. Schmid, A conditional space-time POD formalism for intermittent and rare events: Example of acoustic bursts in turbulent jets, J. Fluid Mech. 867, R2 (2019).

[42] P. Pineau and C. Bogey, Steepened Mach waves near supersonic jets: Study of azimuthal structure and generation process using conditional averages, J. Fluid Mech. 880, 594 (2019).

[43] K. B. M. Q. Zaman, Effect of initial condition on subsonic jet noise, AIAA J. 23, 1370 (1985).

[44] P. J. Morris, The spatial viscous instability of axisymmetric jets, J. Fluid Mech. 77, 511 (1976).

[45] P. J. Morris, The instability of high speed jets, Int. J. Aeroacoust. 9, 1 (2010).

[46] J. B. Freund, S. K. Lele, and P. Moin, Compressibility effects in a turbulent annular mixing layer, Tech. Rep., TF-72, Stanford University, Mechanical Engineering, Flow Physics and Computation Division (1997).

[47] P. Pineau and C. Bogey, Numerical study of temporally-developing supersonic round jets and their sound fields, AIAA Paper 2017-3209 (2017).

[48] C. Bogey, C. Bailly, and D. Juvé, Noise investigation of a high subsonic, moderate Reynolds number jet using a compressible Large Eddy Simulation, Theor. Comput. Fluid Dyn. 16, 273 (2003).

[49] C. Bogey and O. Marsden, Simulations of initially highly disturbed jets with experiment-like exit boundary layers, AIAA J. 54, 1299 (2016).

[50] C. Bogey, Grid sensitivity of flow field and noise of high-Reynolds-number jets computed by large-eddy simulation, Int. J. Aeroacoust. 17, 399 (2018).

[51] C. Bogey and R. Sabatini, Effects of nozzle-exit boundary-layer profile on the initial shear-layer instability, flow field and noise of subsonic jets, J. Fluid Mech. 876, 288 (2019). 
[52] K. Mohseni and T. Colonius, Numerical treatment of polar coordinate singularities, J. Comput. Phys. 157, 787 (2000).

[53] C. Bogey, N. de Cacqueray, and C. Bailly, Finite differences for coarse azimuthal discretization and for reduction of effective resolution near origin of cylindrical flow equations, J. Comput. Phys. 230, 1134 (2011).

[54] C. Bogey and C. Bailly, A family of low dispersive and low dissipative explicit schemes for flow and noise computations, J. Comput. Phys. 194, 194 (2004).

[55] J. Berland, C. Bogey, O. Marsden, and C. Bailly, High-order, low dispersive and low dissipative explicit schemes for multi-scale and boundary problems, J. Comput. Phys. 224, 637 (2007).

[56] C. Bogey and C. Bailly, Influence of nozzle-exit boundary-layer conditions on the flow and acoustic fields of initially laminar jets, J. Fluid Mech. 663, 507 (2010).

[57] C. K. W. Tam and Z. Dong, Radiation and outflow boundary conditions for direct computation of acoustic and flow disturbances in a nonuniform mean flow, J. Comput. Acoust. 4, 175 (1996).

[58] C. Bogey and C. Bailly, Three-dimensional non reflective boundary conditions for acoustic simulations: Far-field formulation and validation test cases, Acta Acust. united Acust. 88, 463 (2002).

[59] A. Michalke, On the inviscid instability of the hyperbolic-tangent velocity profile, J. Fluid Mech. 19, 543 (1964).

[60] A. Michalke, Survey on jet instability theory, Prog. Aerospace Sci. 21, 159 (1984).

[61] R. Sabatini and C. Bailly, Numerical algorithm for computing acoustic and vortical spatial instability waves, AIAA J. 53, 692 (2015).

[62] See the Supplemental Material at http://link.aps.org/supplemental/10.1103/PhysRevFluids.4.124601 for supplemental movies.

[63] K. B. M. Q. Zaman, Flow field and near and far sound field of a subsonic jet, J. Sound Vib. 106, 1 (1986).

[64] R. E. A. Arndt, D. F. Long, and M. N. Glauser, The proper orthogonal decomposition of pressure fluctuations surrounding a turbulent jet, J. Fluid Mech. 340, 1 (1997).

[65] C. Bogey and C. Bailly, Investigation of downstream and sideline subsonic jet noise using large eddy simulations, Theor. Comput. Fluid Dyn. 20, 23 (2006).

[66] G. L. Brown and A. Roshko, On density effects and large structure in turbulent mixing layers, J. Fluid Mech. 64, 775 (1974).

[67] N. Kings and F. Bake, Indirect combustion noise: Noise generation by accelerated vorticity in a nozzle flow, Int. J. Spray Combust. 2, 253 (2010).

[68] H. K. Lee and H. S. Ribner, Direct correlation of noise and flow of a jet, J. Acoust. Soc. Am. 52, 1280 (1972).

[69] J. M. Seiner, The distribution of jet source strength intensity by means of direct correlation technique, Ph.D. thesis, Pennsylvania State University (1974).

[70] J. Panda, Experimental investigation of turbulent density fluctuations and noise generation from heated jets, J. Fluid Mech. 591, 73 (2007).

[71] C. Bogey, S. Barré, D. Juvé, and C. Bailly, Simulation of a hot coaxial jet: Direct noise prediction and flow-acoustics correlations, Phys. Fluids 21, 035105 (2009).

[72] T. Suzuki, Review of diagnostic studies on jet-noise sources and generation mechanisms of subsonicallyconvecting jets, Fluid Dyn. Res. 42, 014001 (2010).

[73] J. Hileman, B. Thurow, E. J. Caraballo, and M. Samimy, Large-scale structure evolution and sound emission in high-speed jets: Real-time visualization with simultaneous acoustic measurements, J. Fluid Mech. 544, 277 (2005).

[74] M. Kearney-Fisher, A. Sinha, and M. Samimy, Intermittent nature of subsonic jet noise, AIAA J. 51, 1142 (2013).

[75] R. Camussi and S. Grizzi, Statistical analysis of the pressure field in the near region of a $M=0.5$ circular jet, Int. J. Aeroacoust. 13, 169 (2014). 